\title{
Improving energy efficiency via probabilistic rate combination in 802.11 multi-rate wireless networks
}

\author{
Yuedong $\mathrm{Xu}^{\mathrm{a}}$, John C.S. Lui ${ }^{\mathrm{a}, *}$, Dah-Ming Chiu ${ }^{\mathrm{b}}$ \\ ${ }^{a}$ Department of Computer Science and Engineering, The Chinese University of Hong Kong, Hong Kong \\ ${ }^{\mathrm{b}}$ Department of Information Engineering, The Chinese University of Hong Kong, Hong Kong
}

\section{A R T I C L E I N F O}

\section{Article history:}

Received 8 December 2007

Received in revised form 20 January 2009

Accepted 27 January 2009

Available online 6 February 2009

\section{Keywords:}

Energy efficiency

Probabilistic rate combination

Rate anomaly

CPT/CWA

Stability

\begin{abstract}
A B S T R A C T
Energy efficiency is one of the most important concerns in wireless networks because wireless clients usually have limited battery power. The aim of this work is to reduce energy consumption by exploiting multi-rate diversity in 802.11 wireless networks. An important observation is that "probabilistic rate combination" in transmission can significantly reduce power consumption. We formulate the energy efficient rate combination as a non-convex optimization problem. A non-cooperative rate adaptation scheme is presented to reduce power consumption without information exchange. Each node selects rate combination strategy and computes its transmission probability based on the weighted average interface queue length. Due to the well-known "rate anomaly" problem, selfish nodes may choose to transmit at a lower rate free ride from the other nodes. To mitigate this problem, we propose a joint consecutive packet transmission (CPT) and contention window adaptation mechanism (CWA). We prove the stability of our proposed algorithm, and to the best of our knowledge, this is the first control theoretical analysis on 802.11 "multi-rate" wireless networks. Simulation results show that the probabilistic rate combination can greatly save battery power, even up to $700 \%$ times compared with standard $802.11 \mathrm{a} / \mathrm{h}$ protocol.
\end{abstract}

Crown Copyright (c) 2009 Published by Elsevier B.V. All rights reserved.

\section{Introduction}

Reducing power consumption is one of the most important issues in wireless networks because prevalent wireless devices, such as handhelds and laptops, still suffer from limited battery capacity. Despite the energy expenditure in the basic operations, communication components like network interface cards consume a lot of battery energy. If one can reduce power consumption of packet transmission, the lifetime of wireless nodes can be extended.

In the past few years, number of methods have been proposed to improve the energy efficiency of 802.11 wireless networks. These methods can be roughly grouped into five classes by adjusting: (1) sleep/awake modes; (2) transmission power; (3) physical layer data rate; (4) random ac-

\footnotetext{
* Corresponding author. Tel.: +852 2609 8442; fax: +852 26035024 .

E-mail addresses: ydxu@cse.cuhk.edu.hk (Y. Xu), cslui@cse.cuhk.edu.hk (J.C.S. Lui), dmchiu@ie.cuhk.edu.hk (D.-M. Chiu).
}

cess mechanism, and (5) routing. The 802.11 has a power saving mode to reduce power consumption, in which a wireless interface can enter the sleep mode, periodically wakes up to receive packets from a neighbor that may have backlogged packets for the sleeping node. However, the switching mechanism of sleep/awake modes cannot reduce the energy during packet transmission. An alternative way to conserve energy is to apply power control [1-4]. A wireless node is allowed to transmit using the minimum power level that can sustain successful transmissions. The advent of $802.11 \mathrm{a} / \mathrm{b} / \mathrm{g} / \mathrm{h}$ multi-rate standards imposes new challenges on the power saving mechanisms. Miser [4], an intelligent power control algorithm, computes an optimal rate-power combination table for $802.11 \mathrm{a} / \mathrm{h}$ in an offline manner, and then at runtime, a wireless station determines the most energy efficient transmission strategy for each data frame via table lookup. Authors in [5] present a heuristic, cooperative rate adaptation algorithm to save power for a given traffic demand in multi-hop ad hoc net- 
works. In this paper, we address the energy conservation problem in 802.11 multi-rate wireless networks. Distinguished from previous work (e.g. [5,4]), we present a distributed algorithm that does not need information exchange and is immunized from consequent information cheating.

The contributions of our work are as follows. First, we investigate the impact of "probabilistic" transmission rate strategy on energy consumption in $802.11 \mathrm{a} / \mathrm{h}$ wireless networks, which has not been studied so far. We have three important observations for the 802.11a/h devices: (1) transmitting at a lower physical data rate does not necessary consume less energy than that of a higher data rate; (2) probabilistic rate combination can remarkably reduce energy consumption; (3) in a CSMA/CA wireless network that contains multiple energy-centric selfish nodes, those with smaller traffic loads might have no incentive to increase their data rates. This is the consequence of the famous "rate anomaly" [8] problem.

Based on these observations, we identify the energy efficient rate combination strategy that can support a given traffic workload and at the same time, achieve energy efficiency. Second, we model the physical data rate combination among competing nodes as a non-convex optimization problem. A joint consecutive packet transmission (CPT) and contention window adaptation (CWA) method is introduced to encourage wireless nodes to support the traffic demand by increasing their physical data rates. Third, we propose a non-cooperative rate adaptation scheme to reduce energy consumption and balance it among all active nodes. The dynamics of interface queue length serves to judge whether the traffic demand is satisfied or not. A proportional controller plus low-pass filter is used to control the transmission probability of using the high data rate in its strategy set. Last, we prove the stability of this non-cooperative method. Simulation results show that the proposed algorithm can remarkably reduce energy expenditure in 802.11a/h wireless networks.

The paper is organized as follows. The relationships among transmission powers, data rates and energy consumption are revealed in Section 2. We formulate the mathematical model of rate adaptation schemes and analyze their properties in Section 3. We propose a non-cooperative combination algorithm to improve energy efficiency in Section 4. In Section 5, we present NS2 simulations for a variety of network scenarios. Related work is presented in 6 . Section 7 concludes.

\section{Revealing the relationship among energy, power and data rate}

\subsection{Motivation}

IEEE $802.11 \mathrm{a} / \mathrm{h}$ standards provide eight physical data rates which require different minimum levels of signal qualities. A lower data rate demands smaller SNR threshold than that of a higher data rate, therefore, it may seem that it is more energy efficient to transmit a data frame using lower data rates. Due to limited modulation schemes in IEEE 802.11a/h, wireless nodes usually have to transmit at a higher data rate than their given traffic demands. The energy consumption problem will be more severe when one considers channel fading or interference from concurrent transmissions. In this section, we first reveal the relationship between energy consumption and rate selection for a single flow in a wireless network. An interesting finding is that probabilistic transmission with different rates can satisfy the traffic demand and at the same time, reduce power consumption. Within the available data rates of 802.11a/h standards, we can identify the optimal rate combination for any feasible traffic demand.

\subsection{Deriving transmission power}

The $802.11 \mathrm{a} / \mathrm{h}$ adopts orthogonal frequency division multiplexing (OFDM) mechanism at the $5 \mathrm{GHz}$ U-NII band and provides only eight data rates with different modulation schemes and coding rates. As shown in Table 1 $[13,6]$, to transmit at a given data rate, the signal to noise ratio (SNR) has to exceed a given threshold.

Using Shannon formula, the achievable channel rate $\Gamma_{c}$ under additive white Gaussian noise (AWGN) channel can be expressed by

$\Gamma_{c}=W \log _{2}(1+S N R)$,

where $W$ is the channel bandwidth in Hertz. Current commercial 802.11 products have limited adaptive coding and modulation schemes and merely support the above eight rate levels.

Denote $\lambda$ to be the speed of light and $f$ to be the center frequency of wireless spectrum. Based on the power propagation model, the path loss at a receiver which is $d$ meters away from the reference distance $d_{0}$ (i.e. $1 \mathrm{~m}$ in a general environment) of the transmitter is

$P_{r x}^{i}=P_{t x}^{i} \cdot\left(\frac{\lambda}{4 \pi f d_{0}}\right)^{2} \cdot\left(\frac{d_{0}}{d}\right)^{\theta}$

where $P_{t x}^{i}$ and $P_{r x}^{i}$ are transmission powers at the transmitter and the signal strength at the receiver when the $i$ th data rate is used. We denote $S N R_{i}^{\text {th }}$ as the minimum SNR requirement for the $i$ th data rate (e.g. $S N R_{1}^{\text {th }}(d B)=6.02 \mathrm{~dB}$ in Table 1), $\theta$ is the path loss exponent and the value has $2 \leqslant \theta \leqslant 5$. Let $P_{N}$ denote the power of additive white Gaussian noise (AWGN). The minimum transmission power to support rate $r_{i}$ is given by

$P_{t x}^{i} \geqslant P_{N} \cdot d^{\theta} \cdot S N R_{i}^{t h} \cdot\left(\frac{4 \pi f}{\lambda}\right)^{2}$.

Table 1

For BERs less than or equal to $10^{-5}$, the minimum SNR required to support the corresponding data rate.

\begin{tabular}{lccl}
\hline Rate Level & Rates $(\mathrm{Mbps})$ & SNR $(\mathrm{dB})$ & Modulation \\
\hline 1 & 6 & 6.02 & BPSK \\
2 & 9 & 7.78 & BPSK \\
3 & 12 & 9.03 & QPSK \\
4 & 18 & 10.79 & QPSK \\
5 & 24 & 17.04 & 16-QAM \\
6 & 36 & 18.80 & 16-QAM \\
7 & 48 & 24.05 & 64-QAM \\
8 & 54 & 24.56 & 64-QAM \\
\hline
\end{tabular}




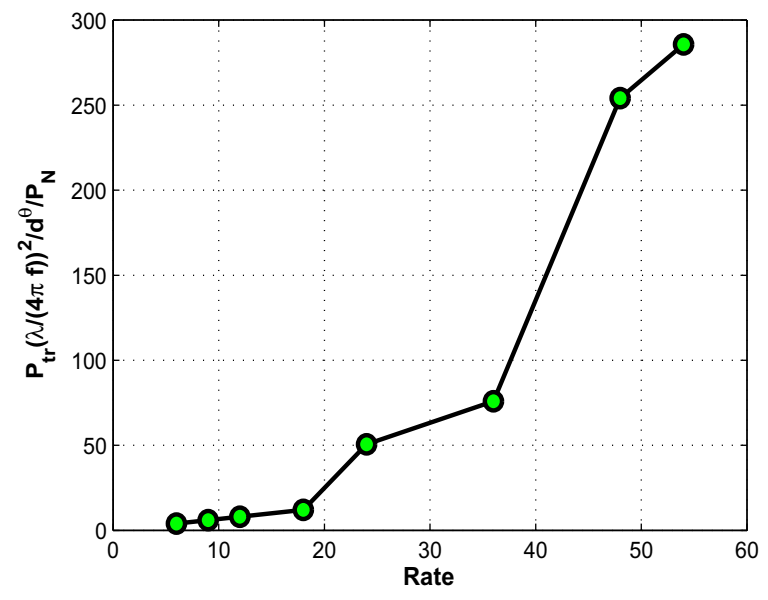

Fig. 1. Power-rate curve.

Because $802.11 \mathrm{a} / \mathrm{h}$ signal strengths have a very wide dynamic range, SNRs are usually expressed in the logarithmic decibel scale. By definition, SNR is 10 times the logarithm of the power ratio. Then, the minimum transmission power can be expressed using the following alternative forms:

$P_{t x}^{i} \geqslant P_{N} \cdot d^{\theta} \cdot 10^{\frac{1}{10} S N R_{i}^{t h}(d B)} \cdot\left(\frac{4 \pi f}{\lambda}\right)^{2}$

or

$$
\begin{aligned}
P_{t x}^{i}(d B) \geqslant & P_{N}(d B)+S N R_{i}^{t h}(d B)+10 \log _{10} d+20 \log _{10} \\
& \times \frac{4 \pi f}{\lambda} .
\end{aligned}
$$

The constant term $\left(\frac{4 \pi f}{\lambda}\right)^{2}$, is denoted to be $P L\left(d_{0}\right)$ where $d_{0}$ is $1 \mathrm{~m}$, and is used only in the computation of energy consumption.

According to the SNR thresholds of available 802.11a/h PHY rates in Table 1, we exhibit the relationship between transmission powers and data rates. One can see in Fig. 1 that a higher data rate requires a higher power level to sustain the successful decoding in the receiver. The minimum transmission powers of those rates with the same modulation scheme are not so far from each other, while the differences of required power levels among various modulation schemes are quite distinct.

\subsection{Deriving energy consumption}

We denote the average power consumption of a node as $\mathscr{E}$, which is the average energy used per-second. $\mathscr{E}$ is composed of energy consumption in both transmit mode and idle mode. Authors in [7] find that the idle mode spends comparable energy to the receive mode. The purpose of this paper is to reduce energy consumption in the transmit mode by choosing some lower rates for the given traffic loads. Lower transmit rates further cut down the idle time of wireless clients, reducing the energy consumption in the idle mode. In this work, we only consider the energy consumption for transmission events. Our simplification is justified due to three major reasons. First, the idle and receiving powers are small compared with the transmission power, especially when wireless nodes transmit using high data rates. Second, the idle power can be saved by switching to sleep mode. The receiving energy is always fixed for a node, and is unimportant when the receiver is an AP with energy supply. Third, we separate transmission-energy reduction method from many other existing schemes so as to highlight our motivation. Denote $P_{t x, o}$ to be the transmission power of the rate-independent overhead and $\bar{P}_{t x}$ to be the average power of data frames, the average energy consumption $\mathscr{E}$ is represented by

$\mathscr{E}=A_{o} P_{t x, o}+A_{p} \bar{P}_{t x}$,

where $A_{o}$ and $A_{p}$ are the normalized airtime of overheads and data packets, respectively. The energy consumption of one data packet $\xi_{\text {data }}$ can be computed by

$\xi_{\text {data }}\left(\ell, r_{i}, P_{t x}\right)=T_{\text {data }}\left(\ell, r_{i}\right) \cdot P_{t x}$,

where $P_{t x}$ can be computed via Eq. (3) or (4), $\ell$ is the payload size, and $T_{\text {data }}\left(\ell, r_{i}\right)$ is the data transmission duration which is given by

$T_{\text {data }}\left(\ell, r_{i}\right)=\ell / r_{i}$.

The energy consumed in sending an ACK frame is

$\xi_{\text {ack }}=T_{a c k}\left(r_{i}^{\prime}\right) \cdot P_{t x}^{a c k}$,

where the PHY rate used for the ACK transmission, $r_{i}^{\prime}$, is chosen to be the highest one in the BSS basic rate set that is less than or equal to the rate of the data frame. In this work, the PHY/MAC overheads are transmitted using $6 \mathrm{Mbps}$ in the BSS uniformity. The transmission power of PHY/MAC overheads is $3 \mathrm{~dB}$ higher than the required level so as to let the overhead packets be received with very small bit error rate. The above equations will be used to calculate the total power expenditure throughout our paper. The airtime and power of PHY/MAC overheads are constants when transmitting a whole MAC frame, their power consumptions are fixed in every transmission, no matter what data rate is used for data payloads. For a given traffic demand, the total number of transmission is almost fixed, resulting the fixed overhead airtime and the fixed

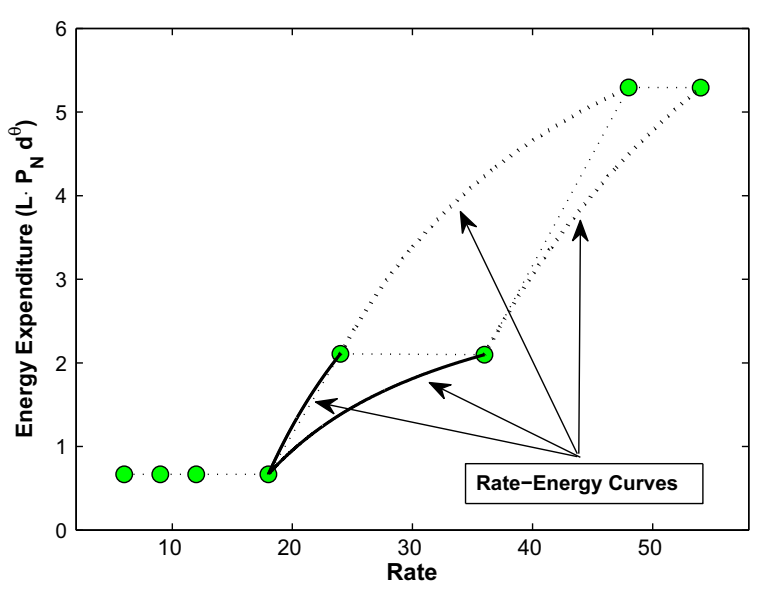

Fig. 2. Energy expenditure vs. rate strategy. 
overhead energy expenditure in a unit time (e.g. $1 \mathrm{~s}$ ). Therefore, in the analysis we only consider energy of data packets, the unspecified part of energy in a transmission. Nevertheless, the energy consumptions of payloads and whole frames are considered separately in the performance evaluation.

It is important to note that a higher data rate only needs a smaller airtime than that of a lower data rate when transmitting a data frame, therefore, a higher data rate does not necessarily consume more energy than a lower one. To illustrate, the eight points in Fig. 2 represent the power consumptions of transmitting a data frame at each rate level and some of them have "comparable" energy expenditures. ${ }^{1}$ It is very likely to waste a lot of energy to support a traffic load without rate adaptation. For example, when the traffic demand is $18.5 \mathrm{Mbps}$ and the transmitter has to select the data rate at $24 \mathrm{Mbps}$ so as to satisfy the demand, but at this data rate, the wireless node consumes more energy than necessary. Obviously, a combination of both low and high data rates can effectively reduce energy consumption.

We introduce a novel probabilistic transmission scheme to save energy for wireless nodes. One interesting finding is that a higher data rate does not necessarily consume more power than that of a lower one. For example, Fig. 2 illustrates that data rates $\{6,9,12,18\}$ Mbps have similar energy consumption. Base on this finding, the selection of transmission rates is not arbitrary, but has an optimal close-form solution. Consider that 802.11 MAC uses a higher rate $r_{j}$ to transmit a frame with probability $\gamma(0 \leqslant \gamma \leqslant 1)$, and uses a lower rate $r_{i}$ with probability $1-\gamma$, the average data rate $x$ can be expressed as

$x=\frac{\ell}{\bar{T}_{\text {data }}\left(\ell, r_{i}, r_{j}\right)}=\frac{\ell}{(1-\gamma) \frac{\ell}{r_{i}}+\gamma \frac{\ell}{r_{j}}}=\frac{r_{i} r_{j}}{\gamma r_{i}+(1-\gamma) r_{j}}$.

The corresponding power consumption of this probabilistic data transmission $\left\{r_{i}, r_{j}\right\}$ can be calculated by

$\xi_{\text {data }}\left(r_{i}, r_{j}, \gamma\right)=(1-\gamma) \xi_{\text {data }}\left(r_{i}\right)+\gamma \xi_{\text {data }}\left(r_{j}\right)$.

In Fig. 2, the curves between two rates represent the relationship between power consumption and average data rate when $\gamma$ increases from 0 to 1 . They serve as examples to illustrate how probabilistic rate combination can satisfy the traffic load and save energy at the same time. We next combine three rates, $r_{i}, r_{j}$ and $r_{k}$ together where there has $r_{i}<r_{j}<r_{k}$. The probability of using $r_{j}$ is $\gamma_{j}$ and that of using $r_{k}$ is $\gamma_{k}$. Then, the average data rate $x$ is represented by

$$
\begin{aligned}
x & =\frac{\ell}{\bar{T}_{\text {data }}\left(\ell, r_{i}, r_{j}, r_{k}\right)}=\frac{\ell}{\left(1-\gamma_{j}-\gamma_{k}\right) \frac{\ell}{r_{i}}+\gamma_{j} \frac{\ell}{r_{j}}+\gamma_{k} \frac{\ell}{r_{k}}} \\
& =\frac{r_{i} r_{j} r_{k}}{\left(1-\gamma_{j}-\gamma_{k}\right) r_{j} r_{k}+\gamma_{j} r_{i} r_{k}+\gamma_{k} r_{i} r_{j}} .
\end{aligned}
$$

Given the transmission probabilities of different data rates, the average power consumption of a packet is expressed as:

$$
\begin{aligned}
\xi_{\text {data }}\left(r_{i}, r_{j}, r_{k}, \gamma_{j}, \gamma_{k}\right)= & \left(1-\gamma_{j}-\gamma_{k}\right) \xi_{\text {data }}\left(r_{i}\right)+\gamma_{j} \xi_{\text {data }}\left(r_{j}\right) \\
& +\gamma_{k} \xi_{\text {data }}\left(r_{k}\right) .
\end{aligned}
$$

Similarly, one can combine more than three data rates together to reduce energy consumption.

However, to utilize the probabilistic rate combination technique, one has to face the following three challenges. First, a natural question is how to choose data rates for probabilistic combination. The $802.11 \mathrm{MAC}$ has eight rate levels so that there are 247 kind of combination strategies in total. A node needs to choose a limited number of rate combination strategy among them. Second, the network capacity is greatly reduced if we naively apply probabilistic rate combination. This subtlety is due to the "rate anomaly" problem [8] in 802.11 since two saturated nodes almost have the equal throughput if their frame sizes are approximately the same, even though their data rates are different. The reason lies in that the 802.11 CSMA MAC guarantees the equal long-term channel access probabilities, instead of the airtime shares for all the competing nodes. For example, if there are two saturated nodes and one node transmits at $6 \mathrm{Mbps}$ and the other node transmits at $54 \mathrm{Mbps}$, then the total system throughput is approximately upper bounded by 12 Mbps, which is lower than the network capacity. Third, nodes with smaller traffic demands may have no incentive to increase their data rates because they can free ride from the rate increase of those nodes with high traffic demands. We will address these problems in Section 3 .

\subsection{Energy efficient rate combination}

After identifying the energy efficiency problem, we need to find an optimal rate combination to support a given traffic demand. Here, we regard that the wireless node can select any level in the available rate set of $802.11 \mathrm{a} / \mathrm{h}$ standards (as illustrated in Table 1). The difference between the minimum power consumption to transmit a data frame among the rate set $\{6,9,12,18\}$ Mbps is very small, and the minimum power consumption of $54 \mathrm{Mbps}$ data rate is comparable to that of $48 \mathrm{Mbps}$. Similar phenomenon also happens when using $24 \mathrm{Mbps}$ and $36 \mathrm{Mbps}$ to transmit a data frame. The power consumption in transmitting a data frame can be computed using Eqs. (4), (7) and (8). In summary, we use the following values without considering the constant term $P L\left(d_{0}\right): \xi_{\text {data }}\left(r_{1}\right):=$ $10^{0.602} / 6 \cdot \ell P_{N} d^{\theta}:=0.666 \cdot \ell P_{N} d^{\theta}:=\quad \xi_{\text {data }}\left(r_{2}\right):=\xi_{\text {data }}\left(r_{3}\right):=$ $\xi_{\text {data }}\left(r_{4}\right) ; \xi_{\text {data }}\left(r_{5}\right):=10^{1.704} / 24 \cdot \ell P_{N} d^{\theta}:=\xi_{\text {data }}\left(r_{6}\right):=2.107$. $\ell P_{N} d^{\theta} ; \quad \xi_{\text {data }}\left(r_{7}\right):=10^{2.405} / 48 \cdot \ell P_{N} d^{\theta}:=\xi_{\text {data }}\left(r_{8}\right):=5.293$. $\ell P_{N} d^{\theta}$.

In order to improve energy efficiency and meet the traffic demand, we need to consider two issues. First, how to select data rates from the available rate set? Second, how to decide the transmission probabilities of different rates? A hybrid rate scheme that contains only two data rates is more appropriate because it introduces only one parameter: $\gamma$. Meantime, two data rates are enough to achieve optimal energy consumption if they are chosen properly. Based on the energy-rate relationship, we have the following proposition. 
Proposition 1. Given a transmitting node with feasible traffic demand C Mbps under an AWGN wireless channel, we have

(1) If the traffic demand is not larger than $18 \mathrm{Mbps}$, any combination of the rate set $\{6,9,12,18\}$ Mbps can achieve the minimum power consumption.

(2) If the traffic demand is within the range $\{18,36\} \mathrm{Mbps}$, the rate combination of $\{18,36\}$ Mbps is optimal for energy efficiency.

(3) If the traffic demand is within the range $\{36,54\} \mathrm{Mbps}$, the rate combination of $\{36,54\}$ Mbps is optimal for energy efficiency.

Proof. Because the item (1) is too simple and item (3) is very close to item (2), we only prove the item (2) in this work.

Assume the traffic demand $C$ is within the range $\{18 M$, $24 M\}$, there are three rate combination schemes (a): $\{18 M$, $24 M\}$, (b): $\{18 M, 36 M\}$ and (c): $\{18 M, 36 M, 54 M\}$. The probabilities of using higher rate are denoted to be $\gamma_{A}$ and $\gamma_{B}$ in scheme (a) and (b), respectively. In the scheme (c), we denote the probabilities of using $36 M$ and $54 M$ to be $\gamma_{C}$ and $\gamma_{D}$, respectively. To begin with, we prove the claim that $\{18 M, 36 M\}$ is the best hybrid transmission scheme if at most two data rates are allowed to combine. According to Eq. (10), there has

$\gamma_{A}=\frac{2 \boldsymbol{C}-36}{\boldsymbol{C}}, \quad \gamma_{B}=\frac{4 \boldsymbol{C}-72}{\boldsymbol{C}}$.

And the energy consumption can be calculated by

$\xi_{\text {data }}\left(r_{4}, r_{6}, \gamma_{A}\right)=\left(1-\gamma_{A}\right) \xi_{\text {data }}\left(r_{4}\right)+\gamma_{A} \xi_{\text {data }}\left(r_{6}\right)$,

$\xi_{\text {data }}\left(r_{4}, r_{5}, \gamma_{B}\right)=\left(1-\gamma_{B}\right) \xi_{\text {data }}\left(r_{4}\right)+\gamma_{B} \xi_{\text {data }}\left(r_{5}\right)$.

Therefore, it is easy to show

$$
\begin{aligned}
& \xi_{\text {data }}\left(r_{4}, r_{5}, \gamma_{B}\right)-\xi_{\text {data }}\left(r_{4}, r_{6}, \gamma_{A}\right) \\
& =\frac{2.8816 C-51.8868}{C} \cdot \ell P_{N} R^{\theta} \geqslant 0 .
\end{aligned}
$$

The above expression is always non-negative for any $\boldsymbol{C}$ satisfying $18 M \leqslant \boldsymbol{C} \leqslant 36 M$ and the sign of equality holds only when the traffic demand $\boldsymbol{C}$ is $18 \mathrm{Mbps}$. Step by step, we can finally prove item (2) for any strategy that at most two data rates are allowed to combine. Next, we show that the strategy $\{18,36\}$ Mbps is better than any one that has at least three rates. Taking scheme (c) as an example, the following equations hold for $\gamma_{C}$ and $\gamma_{D}$

$\boldsymbol{C}=\frac{108}{6-3 \gamma_{C}-4 \gamma_{D}}$ or $\gamma_{C}=\frac{6 \boldsymbol{C}-4 \gamma_{D} \boldsymbol{C}-108}{3 \boldsymbol{C}}$.

The corresponding energy consumption of a data packet is expressed as

$$
\begin{aligned}
\xi_{\text {data }}\left(r_{4}, r_{6}, r_{8}, \gamma_{C}, \gamma_{D}\right)= & \left(1-\gamma_{C}-\gamma_{D}\right) \xi_{\text {data }}\left(r_{4}\right)+\gamma_{C} \xi_{\text {data }}\left(r_{6}\right) \\
& +\gamma_{D} \xi_{\text {data }}\left(r_{8}\right) .
\end{aligned}
$$

The difference of energy expenditure between scheme (a) and $(\mathrm{c})$ is

$$
\begin{aligned}
& \xi_{\text {data }}\left(r_{4}, r_{6}, r_{8}, \gamma_{C}, \gamma_{D}\right)-\xi_{\text {data }}\left(r_{4}, r_{6}, \gamma_{A}\right) \\
& =\left(\gamma_{C}-\gamma_{A}\right)\left(\xi_{\text {data }}\left(r_{6}\right)-\xi_{\text {data }}\left(r_{4}\right)\right)+\left(\xi_{\text {data }}\left(r_{8}\right)-\xi_{\text {data }}\left(r_{4}\right)\right) \gamma_{D} \\
& =-\frac{4 \gamma_{D}}{3}\left(\xi_{\text {data }}\left(r_{6}\right)-\xi_{\text {data }}\left(r_{4}\right)\right)+\left(\xi_{\text {data }}\left(r_{8}\right)-\xi_{\text {data }}\left(r_{4}\right)\right) \gamma_{D} \\
& \approx 2.71 \gamma_{D} \cdot \ell P_{N} R^{\theta}>0,
\end{aligned}
$$

as long as $\gamma_{D}$ is greater than 0 . Clearly, one can see that scheme (a) is better than scheme (c). Using the similar method, we can easily prove the superiority of scheme (a) over other rate combination strategies.

In summary, there are two efficient rate strategies to reduce energy consumption in a wireless channel with free path power loss. According to the given traffic demand, a node can choose rates from the set $\{18,36\} \mathrm{Mbps}$ and $\{36,54\}$ Mbps. Note that in some practical 802.11 devices, the SNR thresholds might not exactly follow the values in Table 1. Under this situation, the optimal rate combination might not always be achieved by a strategy with two or three rates. But with the above method, one can always find an efficient strategy to satisfy the traffic demand and reduce energy consumption simultaneously.

\subsection{The impact of PHY/MAC overheads and binary backoff}

The transmission of PHY overheads is payload-rate independent. In other words, the 802.11a/h device uses the basic rate (i.e. $6 \mathrm{Mbps}$ ) to transmit the PLCP Preamble and the PLCP header. The characteristics of the $802.11 \mathrm{a} / \mathrm{h}$ PHY/MAC are listed in Table 2.

The PHY/MAC overheads are transmitted using the basic rate, but with the maximum power in the default setting. We denote $\xi_{\text {overhead }}$ to be the energy consumption of overheads of a node in each transmission. The PHY/MAC overheads of different wireless nodes are also different. The overheads in the transmitter include the MAC frame head and the corresponding PLCP preamble as well as the PLCP header. The overheads of the receiver contain the ACK frame, the PLCP preamble and the PLCP header. Since the transmission of overheads usually occupies $20-40 \%$ or more of airtime, their energy consumptions could be very high when the overhead power is large. Later on, we experimentally show that the high overhead transmission power leads to remarkable energy waste. Hence, to improve energy efficiency, we recommend to choose large payload size and the small transmission power for PHY/MAC overheads.

RTS/CTS is designed to ameliorate the hidden terminal problems. In order to silence all the hidden terminals, the RTS/CTS should be transmitted at the 6 Mbps using the

Table 2

The 802.11a PHY/MAC characteristics.

\begin{tabular}{lll}
\hline Characteristics & Value & Annotation \\
\hline SlotTime & $9 \mu \mathrm{s}$ & Slot time \\
SIFSTime & $16 \mu \mathrm{s}$ & SIFS time \\
DIFSTime & $34 \mu \mathrm{s}$ & DIFS $=$ SIFS $+2 \times$ Slot \\
$C W_{\min }$ & 15 & Minimum contention window \\
$C W_{\max }$ & 1023 & Maximum contention window \\
PLCPPreamble & $16 \mu \mathrm{s}$ & PLCP preamble duration \\
PLCP-SIG & $4 \mu \mathrm{s}$ & PLCP signal duration \\
OFDM symbol & $4 \mu \mathrm{s}$ & OFDM symbol interval \\
\hline
\end{tabular}


maximum transmission power. Thus, the energy consumptions of RTS/CTS frames are even greater than those of DATA/ACK frames, and is disabled in WLANs and single-cell ad hoc networks. Under 802.11 DCF scheme, the initial contention window (CW) size determines the occupied airtime of a wireless node in the presence of competing nodes. The average transmit rate of a node is expressed by the traffic load over its airtime. Thus, the initial contention window size indirectly determines the energy consumption of a node when transmitting the given traffic load.

\subsection{Rate combination and power control in a fading channel}

From the above analysis, we derive the optimal rate combination for a single flow in an ideal AWGN wireless channel. For example, the rate strategy $\{36 M, 54 M\}$ is more energy efficient than $\{36 M, 48 M\}$ when the traffic demand is within the range $\left\{\frac{36}{\ell+36 e 6\left(T_{o}+T_{b}\right)} M, \frac{48}{\ell+48 e 6\left(T_{o}+T_{b}\right)} M\right\}$. However, path loss, channel fading and interference cause variations in the received signal strength in mobile ad hoc networks. Such variations result in the variations in the BER (bit error rate), which might cause considerable transmission failures. Thus, a natural question is that whether we need to increase the transmission power or adjust the rate combination strategy in the presence of channel fading. Various rate adaptation schemes, such as [14,16,18,15,17], have been introduced to reduce the frame loss due to channel fading or interference in the past several years. These rate adaptation schemes are all focused on the throughput enhancement and based on the vital premise of fixed transmission power for all data rates. But in our rate combination scheme, the transmission powers of different rates are quite different. So, we can apply power control for each rate level based on infrequent channel feedback until the maximum power is reached.

Denote the noise level at the receiver to be $P_{N}$ and the transmission power levels for each rate to be $P_{t x, i},(i=1,2, \ldots, 8)$ at the transmitter under AWGN channel. The minimum received signal strength for each rate level can be represented by $P_{r x, i},(i=1,2, \ldots, 8)$. Due to the channel fading, the free path loss model in Eq. (2) is no longer applicable. Furthermore, a wireless node is also blind to the fading models (e.g. Rician, Raleigh or Nakagami fadings). Hence, the frame loss ratio is the only feasible method to evaluate channel fading. Thanks for the existing power control methods such as $[1,3,2,4]$, we can balance the frame loss ratio and energy consumption for an explicit data rate in a single-cell wireless network. This is to say, one can find a new set of transmission power $P_{t x, i}^{f},(i=1,2, \ldots, 8)$ in a fading channel to optimize the energy consumption. Under the proper transmission power control (TPC), the stationary (average) frame loss ratio for each rate can be denoted to be $\delta_{i}^{f},(i=1,2, \ldots, 8)$. we calculate the effective data rate of the $i$ th rate level $r_{i}^{f}$ and its power consumption for one successful full frame $\xi_{\text {frame }}^{f}\left(r_{i}, \delta_{i}^{f}\right)$ by

$r_{i}^{f}=\left(1-\delta_{i}^{f}\right) \cdot r_{i}$,

$\xi_{\text {frame }}^{f}\left(r_{i}, \delta_{i}^{f}\right)=\frac{\left(\xi_{\text {overhead }}+\xi_{\text {data }}\left(r_{i}, P_{t x, i}^{f}\right)\right)}{1-\delta_{i}^{f}}$.
Our analysis shows that the blue points in Fig. 2 move northwestwards when the stationary frame loss ratios increase along with more and more serious fading. When the largest rate level (i.e. $54 \mathrm{Mbps}$ ) cannot be sustained even using the maximum transmission power $P_{t x}^{\max }$, the rate combination $\{36,54\} \mathrm{Mbps}$ is not energy efficient. Therefore, the MAC layer should switch the strategy from $\{36$, $54\}$ Mbps to $\{36,48\}$ Mbps and label the invalidity of the data rate $54 \mathrm{Mbps}$. The basic idea of this strategy switching is similar to the simple robust rate adaptation algorithm (RRAA) in [15], but we eliminate the inefficient nash equilibria of the original RRAA through consecutive packet transmission and contention window control schemes that will be investigated in the next section.

\section{Mathematical model for rate combination}

In this section, we present the mathematical model as well as theoretical properties of the rate adaptation problems for multiple competing nodes.

\subsection{Mathematical model}

Consider a single-cell wireless network that contains $N$ independent transmission pairs. The traffic demands are described by $C_{n}$ (in unit of bps), for $n=1,2, \ldots, N$. The data rate of node $n$ is denoted to be $x_{n}$. Denote $T_{o}$ and $\bar{T}_{b}$ to be the overhead airtime and the average backoff time of a MAC frame, respectively. Assume that the network capacity can sustain the traffic demands, the system-wide objective function is to minimize the aggregate energy consumption, that is

$$
\begin{aligned}
\text { minimize } & \sum_{n=1}^{N} \frac{C_{n}}{\ell} \cdot\left(\xi_{\text {data }}^{n}\left(x_{n}\right)+\xi_{\text {overhead }}^{n}\right), \\
\text { subjectto } & \sum_{n=1}^{N} \frac{C_{n}}{x_{n}} \leqslant 1-\left(T_{o}+\bar{T}_{b}\right) \cdot \sum_{n=1}^{N} \frac{C_{n}}{\ell}, \\
& x_{n} \in\{6,9,12, \ldots, 54\}, \quad \forall n=1,2, \ldots, N,
\end{aligned}
$$

where $\xi_{\text {data }}^{n}\left(x_{n}\right)$ is the energy consumption per-frame with rate $x_{n}, C_{n} / \ell$ and $C_{n} / x_{n}$ are the number of frames per-second and the channel utilization for the $n$th transmitting node. Since the probabilistic rate combination is not used, the data rate $x_{n}$ can only be chosen from the set $\{6,9,12, \ldots, 54\} \mathrm{Mbps}$. To find the optimal transmission rates for different nodes, one will have to use exhaustive search method which is of high computational complexity.

Another difficulty originates from the challenges of practical implementation. To enable the centralized search, every transmitting node needs the following knowledge of other nodes: average backoff window, traffic rate, frame length as well as collision loss. This centralized method also needs frequent information exchanges such as needed airtime and energy expenditure, which might not be scalable for ad hoc mode. Furthermore, information exchange incurs a serious security problem that malfunctions the rate selection procedure. More specifically, a malicious node reports forged energy expenditures to the AP or to other nodes so as to require low data rate to save the real energy expenditure. Aiming at these challenges, we propose to re- 


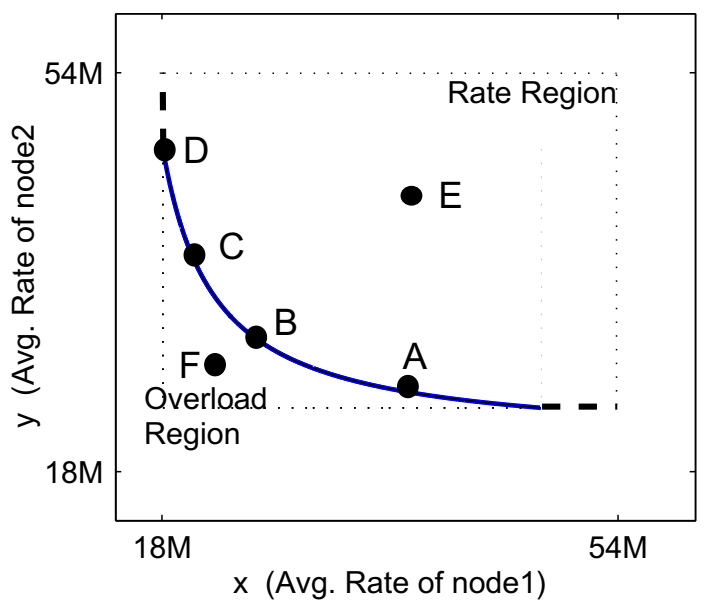

Fig. 3. Converge to an inefficient point.

duce energy consumption by using heuristic probabilistic rate combination in a distributed way. However, a naive application of probabilistic rate combination leads to selfish behavior in the presence of competing users in a cell. For the purpose of saving energy, wireless nodes intend to choose some low rates, which makes the network saturated even when their traffic loads are not high. Due to the "rate anomaly" problem of saturated users, those with small traffic loads have no incentive to increase their average data rates. We illustrate this selfishness via a case study of two competing links in the following subsection.

\subsection{Case studies of two source-destination pairs}

The 802.11 medium access method may cause serious performance problems if it works together with probabilistic rate combination. On one hand, nodes transmit at lower rates may decrease the network capacity. Also, nodes with smaller traffic demands have no incentive to increase their data rates. Let us illustrate the problem through a case study with two transmission pairs without considering PHY/MAC overheads. Let the traffic loads of these two flows to be $C_{1}$ and $C_{2}$ where $C_{1}<C_{2}$ and $18 \leqslant C_{1}+C_{2} \leqslant 54$. Suppose that each transmitting node adjusts its average rate so as to meet its traffic demand. The worst case is that one of the traffic demand cannot be transmitted due to the "rate anomaly" problem. Now let us look at the selfish behavior when both traffic demands can be satisfied. The curve in Fig. 3 represents the trajectory $\frac{C_{1}}{x}+\frac{C_{2}}{y}=1$ where $x$ and $y$ are the average data rates of node 1 and node 2 . The physical meaning of this equation is that a rate strategy along this curve can transmit the given demands and fully utilize the channel at the same time. A point $\left(x_{E}, y_{E}\right)$ above the curve means that the channel is not fully utilized. Correspondingly, a point $\left(x_{F}, y_{F}\right)$ cannot satisfy the given traffic demands. Let the initial rate strategy be $\left(x_{A}, y_{A}\right)$. Because $C_{1}<C_{2}$ and $x_{A} y_{A}$, node 1 will choose a smaller rate $x_{B}$ for the purpose of reducing power consumption. Since their saturated throughput are the same and $C_{1}<C_{2}$, node 2 is more liable to be congested and has to choose a rate $y_{B}$. However, the benefit from the
Table 3

Comparison for CPT, CWA and CPT/CWA.

\begin{tabular}{llllllllll}
\hline & Rates & 6 & 9 & 12 & 18 & 24 & 36 & 48 & 54 \\
\hline CWA & $C W_{\min }$ & 144 & 96 & 72 & 48 & 36 & 24 & 18 & 16 \\
CPT & Packets & 1 & 1.5 & 2 & 3 & 4 & 6 & 8 & 9 \\
CPT/CWA & $C^{2} W_{\min }$ & 48 & 32 & 24 & 16 & 16 & 16 & 16 & 16 \\
& Packets & 1 & 1 & 1 & $\mathbf{1}$ & $\frac{4}{3}$ & $\mathbf{2}$ & $\frac{8}{3}$ & 3 \\
\hline
\end{tabular}

rate increase of node 2 is also shared by node 1 , node 1 further reduces its average rate along the curve until the lowest rate that can sustain $C_{1}$. Eventually node 1 and node 2 will choose the rate strategy $\left(x_{D}=18 \mathrm{Mbps}, y_{D}=\right.$ $C_{2} /\left(1-\frac{C_{1}}{18}\right)$ ). The inefficient rate selection potentially leads to energy waste in wireless networks, especially when the links with higher loads have longer hop distances.

\subsection{Necessity of consecutive packet transmission (CPT) and contention window adaptation (CWA)}

According to the above analysis, one can see that "rate anomaly" is inherent in 802.11 medium access, which guarantees equal long-term channel access probability, instead of equal airtime share, to all saturated nodes. The 802.11 MAC has two basic methods to address this problem. One is to set different initial contention window sizes for different data rates, namely contention window adaptation (CWA). The other is to let high data rates transmit several packets without contention, namely consecutive packet transmission (CPT). The fundamental principle is rather simple. The saturated throughput of a transmitting node is roughly proportional to the number of packets transmitted consecutively without contention, and inverse proportional to the initial contention window size, $C W_{\min }$. By incorporating the virtues of both methods, we present a joint contention window adaptation (CWA) and consecutive packet transmission (CPT) mechanism. On one hand, we adopt CPT for data rates higher than 18 Mbps so that a node is able to transmit several packets back-to-back when it occupies the channel. On the other hand, we adopt larger initial contention window (CW) sizes for those rates lower than $18 \mathrm{Mbps}$. The parameter comparison of the pure CPT, pure CWA and joint CPT/CWA are shown in Table 3. In the joint CPT/CWA scheme, the quotient $C P T / C W_{\min }$ is proportional to the data rate. Later on, we will analytically show that the throughput ratio of any two nodes is proportional to their average data rate ratio when using these parameters in the CPT/CWA scheme. The fractional consecutive packet transmission such as $4 / 3$ for $24 \mathrm{Mbps}$ represents that a node transmits two contention-free packets with probability $1 / 3$ and one packet with probability $2 / 3$. Note that $C W_{\min }$ is 16 and consecutive packet is 1 for all data rates in the standard DCF scheme. The reasons of using a joint CPT/CWA scheme instead of a pure CPT or a pure CWA scheme are as follows.

- If pure CWA scheme is used, the backoff window sizes are rather large for low data rates. Hence, airtime resource might be wasted in the countdown state of contention window when the number of competing nodes is not large. 
- If pure CPT scheme is adopted, it will induce short-term throughput unfairness for competing nodes. In some cases, a node with high data rate might not have enough packets to transmit, resulting in the waste of airtime that belongs to this node.

- The joint CPT/CWA scheme has the benefit of fair airtime assignment and lessens the drawbacks mentioned above.

\subsection{Theoretical analysis}

Let us first derive the rate-energy curves for rate combination under the CPT/CWA scheme. Note that different initial $C W_{\text {min }}$ does not influence the energy consumption in each transmission, but the consecutive transmission scheme is different because a node can transmit several packets without contention once it grasps the channel. Assume that the current rate combination strategy is $\left\{r_{i}, r_{j}\right\}$, a wireless node transmits $m$ consecutive packets using rate $r_{i}$, and transmits $n$ packets consecutively in each round when using rate $r_{j}$ (e.g. $m=1, n=2$ for $\{18,36\}$ Mbps in Table 3 ). Denote $\gamma^{\prime}$ to be the probability using rate $r_{j}$ and the payload size to be $\ell$, the average payload size in each transmission is $\ell^{\prime}=\left(1-\gamma^{\prime}\right) m \ell+\gamma^{\prime} n \ell$, and the corresponding average transmission time is $\bar{T}_{\text {data }}\left(\ell, r_{i}, r_{j}, \gamma^{\prime}\right)=$ $\frac{n \gamma^{\prime} \ell}{r_{j}}+\frac{\left(1-\gamma^{\prime}\right) m \ell}{r_{i}}$. Then, one can compute the average data rate and the average power consumption of one frame by:

$x=\frac{\ell^{\prime}}{\bar{T}_{\text {data }}\left(\ell, r_{i}, r_{j}, \gamma^{\prime}\right)}=\frac{\left(m\left(1-\gamma^{\prime}\right)+n \gamma^{\prime}\right) r_{i} r_{j}}{m\left(1-\gamma^{\prime}\right) r_{j}+n \gamma^{\prime} r_{i}}$,

$\xi_{\text {data }}\left(r_{i}, r_{j}, \gamma^{\prime}\right)=\frac{n \gamma^{\prime} \xi_{\text {data }}\left(r_{j}\right)+m\left(1-\gamma^{\prime}\right) \xi_{\text {data }}\left(r_{i}\right)}{n \gamma^{\prime}+m\left(1-\gamma^{\prime}\right)}$.

Comparing the rate combination schemes with and without CPT/CWA, we have the following proposition,

Proposition 2. The energy-rate curves of probabilistic rate combination with and without CPT/CWA are equivalent.

Proof. In the probabilistic rate combination without CPT/ CWA, the probability $\gamma$ is a function of the average rate $x$, that is, $\gamma=\frac{r_{j}\left(x-r_{i}\right)}{x\left(r_{j}-r_{i}\right)}$. Thus, the average energy consumption of a data frame can be expressed by:

$\xi_{\text {data }}\left(r_{i}, r_{j}, \gamma, x\right)=\frac{r_{i}\left(r_{j}-x\right) \xi_{\text {data }}\left(r_{i}\right)+r_{j}\left(x-r_{i}\right) \xi_{\text {data }}\left(r_{j}\right)}{x\left(r_{j}-r_{i}\right)}$

Similarly, the probability $\gamma^{\prime}$ is also a function of the average rate $x$ when CPT/CWA is used and $\gamma^{\prime}=\frac{m r_{j}\left(x-r_{i}\right)}{m r_{j}\left(x-r_{i}\right)+n r_{i}\left(r_{j}-x\right)}$. Replacing $\gamma^{\prime}$ in Eq. (18), we have

$\xi_{\text {data }}\left(r_{i}, r_{j}, \gamma^{\prime}, x\right)=\frac{r_{i}\left(r_{j}-x\right) \xi_{\text {data }}\left(r_{i}\right)+r_{j}\left(x-r_{i}\right) \xi_{\text {data }}\left(r_{j}\right)}{\chi\left(r_{j}-r_{i}\right)}$

Hence, Proposition 2 follows:

Remark 1. The analysis in the preceding section shows that $\{18,36\}$ Mbps and $\{36,54\}$ Mbps are efficient rate sets for rate combination, and in Table 3 , the CPT ratio $\frac{n}{m}$ is exactly the ratio of $\frac{r_{j}}{r_{i}}$ (e.g. $m=1$ for $18 \mathrm{Mbps}$ and $n=2$ for $36 \mathrm{Mbps}$ ). Thus, the average data rate in Eq. (17) can be simplified as, $x=\left(1-\gamma^{\prime}\right) r_{i}+\gamma^{\prime} r_{j}$.

Next, we investigate the theoretical throughput of wireless nodes in a single-cell multi-rate wireless network. In order to simplify the analysis, we make the following assumptions.

(A1) Each node only has one flow. For the case of multiple flows in a single node, we simply regard them as a single big flow.

(A2) The collision probability of a packet transmitted by each station is constant and independent, regardless of the number of retransmissions already suffered.

(A3) The traffic demands are large enough to saturate all competing nodes.

(A4) In DCF with CPT, only the first MAC packet data unit (MPDU) within each CPT burst may collide with MPDUs from some other stations. Therefore, the CPT burst packets can be treated as the extended MAC frames after the successful transmission of the first MPDU. Consider a single-cell wireless network that consists of an access point, $M$ active nodes with rate strategy $\left\{r_{4}=\right.$ $\left.18, r_{6}=36\right\}$ and $(N-M)$ active nodes with rate strategy $\left\{r_{6}=36, r_{8}=54\right\}$. Their hybrid transmission probabilities are denoted to be $\gamma_{i}$, for $i=1,2, \ldots, M, \ldots, N$.

It has been shown in [9] that, under the decoupling assumption introduced by Bianchi in [10], the attempt probability of node $i$ for a given collision probability $p_{i}$ is,

$G_{i}\left(p_{i}\right):=\frac{1+p_{i}+\cdots+p_{i}^{K_{i}}}{b_{i, 0}+p_{i} b_{i, 1}+\cdots+p_{i}^{K_{i}} b_{i, K}}$,

where $K_{i}$ is denoted as the maximum number of MAC retries of node $i$ and $b_{i, k}$ is the mean backoff slots at the $k$ th attempt for a frame. Obviously, one can see that the collision probability of node $i$ in a single-cell is independent of the data rates of any competing node in a slot. Let $\boldsymbol{p}$ be the vector of collision probabilities of each node. The collision probability of a node is determined by the attempt probabilities of all saturated nodes based on the slotted model and decoupling assumption [10], that is $p_{i}=f_{i}\left(\beta_{1}, \beta_{2}, \ldots, \beta_{n}\right)=1-\prod_{i=1, j \neq i}^{N}$ $\left(1-\beta_{j}\right)$, where the attempt probability $\beta_{j}=G_{j}\left(p_{j}\right)$. Combining the expressions of all $N$ nodes, we obtain the following multi-dimensional fixed point equations $\boldsymbol{p}=\boldsymbol{f}(\boldsymbol{G}(\boldsymbol{p}))$. Solving the fixed point equations through the method introduced in [9], we obtain the statistical slotted channel status for each node in the cell.

Denote the probabilities of successful transmission, collision and idle slot to be $p_{s, i}, p_{c, i}$ and $p_{d}$, respectively in a heterogeneous wireless network, we have

$$
\begin{aligned}
& p_{s, i}=\beta_{i} \prod_{j=1, j \neq i}^{N}\left(1-\beta_{i}\right), \\
& p_{d}=\prod_{i=1}^{N}\left(1-\beta_{i}\right), \\
& p_{c, i}=1-\sum_{j=1}^{N} p_{s, j}-p_{d} .
\end{aligned}
$$

The successful packet transmission time for node $i$ with actual data rate $r_{x}$ can be represented by $T_{i}^{s}\left(\ell_{i}, r_{x}\right)=\frac{\ell_{i}}{r_{x}}+T_{o}$ (in 
unit of slots). Because we select the higher data rate in the strategy set $\left\{r_{x}, r_{y}\right\}$ with probability $\gamma_{i}$, the average transmission time, in the long run, can be expressed as

$\bar{T}_{i}^{s}\left(\ell_{i}, r_{x}, r_{y}, \gamma_{i}\right)=\bar{T}_{i}^{s}\left(r_{x}, r_{y}\right)=\left(1-\gamma_{i}\right) \frac{\ell_{i}}{r_{x}}+\gamma_{i} \frac{\ell_{i}}{r_{y}}+T_{o}$.

Assume that the frame size of all flows are identical and $\gamma_{i}$ is fixed, we rewrite the symbol $\bar{T}_{i}^{s}\left(\ell_{i}, r_{x}, r_{y}, \gamma_{i}\right)$ as $\bar{T}_{i}^{s}\left(r_{x}, r_{y}\right)$ in a compact form. The average collision time $\bar{T}_{i}^{c}$ is assume to be the longest transmission time of collided packets plus SIFS and DIFS frame intervals if RTS/CTS is disabled. Following the methodology in [10], we obtain $X_{j}$, the longterm throughput of node $j$ without the CPT/CWA mechanism,

$X_{j}=\frac{p_{s, j} \cdot \ell_{j}}{p_{d} T_{s l o t}+\sum_{i=1}^{M} p_{s, i} \bar{T}_{i}^{s}\left(r_{4}, r_{6}\right)+\sum_{i=M+1}^{N} p_{s, i} \bar{T}_{i}^{s}\left(r_{6}, r_{8}\right)+\sum_{i=1}^{N} p_{c, i} \bar{T}_{i}^{c}}$.

Remark 2. One can observe that the "rate anomaly" phenomenon in the probabilistic rate combination scheme. A fast node (i.e. with large average data rate) has approximately the same throughput as a slow node.

When the CPT/CWA schemes are taken into consideration, the CPT burst packets can be regarded as the extended MAC frames after a successful MPDU transmission. The average successful transmission time using $\left\{r_{4}, r_{6}\right\}$ and $\left\{r_{6}, r_{8}\right\}$ can be calculated by,

$$
\begin{aligned}
\overline{T_{i}^{\prime}}\left(r_{4}, r_{6}\right) & =\left(1-\gamma_{i}\right)\left(\ell_{i} / r_{4}+T_{o}\right)+\left(2 \gamma_{i} \ell_{i} / r_{6}+T_{o}\right) \\
& =\ell_{i} / r_{4}+T_{o},
\end{aligned}
$$

${\overline{T^{\prime}}}_{i}^{s}\left(r_{6}, r_{8}\right)=\left(1-\gamma_{i}\right)\left(2 \ell_{i} / r_{6}+T_{o}\right)+\gamma_{i}\left(3 \ell_{i} / r_{8}+T_{o}\right)$

$$
=\ell_{i} / r_{4}+T_{0} \text {. }
$$

With consecutive transmission, collision mainly happens in the first packet. When the maximum number of MAC retries is reached, the 802.11 MAC will cancel the consecutive transmission. The collision time is thus equivalent to the transmission time of one MAC frame. Therefore, we obtain the throughput of node $j,(1 \leqslant j \leqslant M)$ if it chooses the hybrid rate scheme $\left\{r_{4}, r_{6}\right\}, X_{j}=$

$$
\begin{aligned}
& \frac{p_{s, j} \cdot \ell_{j}\left(1+\gamma_{j}\right)}{p_{d} T_{s l o t}+\sum_{i=1}^{M} p_{s, i}{\overline{T^{\prime}}}_{i}^{s}\left(r_{4}, r_{6}\right)+\sum_{i=M+1}^{N} p_{s, i} \bar{T}_{i}^{\prime} s\left(r_{6}, r_{8}\right)+\sum_{i=1}^{N} p_{c, i} \bar{T}_{i}^{c}} \\
& =\frac{p_{s, j} \cdot \ell_{j}\left(1+\gamma_{j}\right)}{p_{d} T_{s l o t}+\sum_{i=1}^{N} p_{s, i}\left(\ell_{i} / r_{4}+T_{o}\right)+\sum_{i=1}^{N} p_{c, i} \bar{T}_{i}^{c}} \\
& =\frac{p_{s, j} \cdot \ell_{j}\left(1+\gamma_{j}\right) r_{4}}{\left(p_{d} T_{\text {slot }}+\sum_{i=1}^{N} p_{s, i}\left(\ell_{i} / r_{4}+T_{o}\right)+\sum_{i=1}^{N} p_{c, i} \bar{T}_{i}^{c}\right) r_{4}} \\
& =\frac{p_{s, j} \cdot \ell_{j} \cdot x_{j}}{\left(p_{d} T_{s l o t}+\sum_{i=1}^{N} p_{s, i}\left(\ell_{i} / r_{4}+T_{o}\right)+\sum_{i=1}^{N} p_{c, i} \bar{T}_{i}^{c}\right) r_{4}}
\end{aligned}
$$

If the node $j$ chooses the hybrid rate scheme $\left\{r_{6}, r_{8}\right\}$ instead, the throughput expression of node $j$ is computed by $X_{j}=$

$$
\begin{gathered}
\frac{p_{s, j} \cdot \ell_{j}\left(2+\gamma_{j}\right)}{p_{d} T_{\text {slot }}+\sum_{i=1}^{M} p_{s, i} \overline{T^{\prime} s}{ }_{i}\left(r_{4}, r_{6}\right)+\sum_{i=M+1}^{N} p_{s, i} \bar{T}_{i}^{\prime} s\left(r_{6}, r_{8}\right)+\sum_{i=1}^{N} p_{c, i} \bar{T}_{i}^{c}} \\
=\frac{p_{s, j} \cdot \ell_{j}\left(2+\gamma_{j}\right)}{p_{d} T_{s l o t}+\sum_{i=1}^{N} p_{s, i}\left(\ell_{i} / r_{4}+T_{o}\right)+\sum_{i=1}^{N} p_{c, i} \bar{T}_{i}^{c}} \\
=\frac{p_{s, j} \cdot \ell_{j} \cdot\left[r_{6}\left(1-\gamma_{j}\right)+r_{8} \gamma_{j}\right]}{\left(p_{d} T_{\text {slot }}+\sum_{i=1}^{N} p_{s, i}\left(\ell_{i} / r_{4}+T_{o}\right)+\sum_{i=1}^{N} p_{c, i} \bar{T}_{i}^{c}\right) r_{4}} \\
=\frac{p_{s, j} \cdot \ell_{j} \cdot x_{j}}{\left(p_{d} T_{s l o t}+\sum_{i=1}^{N} p_{s, i}\left(\ell_{i} / r_{4}+T_{o}\right)+\sum_{i=1}^{N} p_{c, i} \bar{T}_{i}^{c}\right) r_{4}} .
\end{gathered}
$$

Authors in [9] have shown that the successful transmission probability of a node is decided by the average contention window vector $\beta$ in a single-cell. That is, the $p_{d}, p_{s, i}, p_{c, i}$ are independent of PHY rates. Consider two nodes $\mu$ and $v$ using rate set $\left\{r_{4}, r_{6}\right\}$, their throughput ratio $\frac{X_{\mu}}{X_{v}}=\frac{1+\gamma_{\mu}^{\prime}}{1+\gamma_{v}^{\prime}}$ according to Eqs. (26) and (27). Because $r_{6}$ is twice that of $r_{4}$, the average data rates have $x_{\mu}=\left(1+\gamma_{\mu}^{\prime}\right) r_{4}$ and $x_{v}=\left(1+\gamma_{v}^{\prime}\right) r_{4}$, which are derived from Eq. (21). Therefore, the throughput ratio of two nodes is exactly their average data rate ratio, i.e. $\frac{x_{\mu}}{X_{v}}:=\frac{x_{\mu}}{x_{v}}$. Similarly, if node $v$ switches to the rate set $\left\{r_{6}, r_{8}\right\}$, we also have $\frac{X_{\mu}}{X_{v}}:=\frac{x_{\mu}}{x_{v}}$. One can also find that the above result holds for any rate combination when using the CPT/CWA parameters in Table 3.

Remark 3. Using the CPT/CWA scheme, we can make pernode throughput proportional to their physical data rates. In the energy efficient rate adaptation system, a node transmitting at a higher data rate obtains higher throughput. Therefore, each node has the incentive to adapt data rate so as to satisfy its traffic demand.

\section{Non-cooperative rate adaptation}

In this section, we present a non-cooperative rate combination scheme to reduce power consumption in wireless networks. The basic idea is to use queue length based controller to adjust combination strategy and transmit probability. The underline principle is that the change of average interface queue (IFQ) length reflects the rate difference between traffic demand and throughput. We model the noncooperative rate adaptation as a feedback control system and prove its stability.

\subsection{Main idea}

The main idea of non-cooperative rate combination scheme is as follows: when the traffic loading is greater than the node throughput, queue will be built up in the buffer. If the queue size is large, the 802.11 MAC can select a higher probability to transmit data frames consecutively using high data rates. Otherwise, the MAC layer will choose a larger probability to transmit with low data rates. The proposed method is inspired by the active queue management (AQM) mechanism in the wired Internet such as the random early detection (RED) [11] algorithm. The key difference is that the AQMs control the source sending rates, while our scheme adjusts the link bandwidth. 
The 802.11 MAC layer adopts a low-pass filter to calculate the average queue size. Hence, the short-term increases in the queue size that result from bursty traffic or from transient MAC layer congestion do not result in a significant increase in the average queue size. The average queue length $\bar{q}$ can be represented by

$\bar{q}((k+1) \delta)=(1-\omega) \bar{q}(k \delta)+\omega q((k+1) \delta)$,

where $q((k+1) \delta)$ is the sampled instantaneous queue length. The EWMA parameter, $\omega \in[1]$, determines the time constant of the low-pass filter. The transmission probability $\gamma$ is computed by comparing the average queue length with two thresholds: a minimum threshold and a maximum threshold. When the average queue size is less than the minimum threshold and the incoming rate is no larger than the average throughput, the MAC layer switches the combination scheme to a strategy set of lower rates. When the average queue size is greater than the maximum threshold and the average traffic demand is also larger than the average throughput, the 802.11 MAC upgrades to the rate combination scheme with higher rates. To avoid fluctuation of switching behavior, we introduce a freeze timer so that consecutive switchings are not allowed in a short time interval.

Next, we explain the non-cooperative scheme to determine the transmission probability $\gamma$ when the combination scheme is fixed. Consider the current rate combination scheme $\left\{r_{x}, r_{y}\right\}$ where $r_{y} r_{x}$, we obtain the transmission probability of using data rate $r_{y}$ as,

$\gamma= \begin{cases}0 & \text { if } \bar{q}(k \delta) \leqslant q_{\min }, \\ \frac{\bar{q}(k \delta)-q_{\min }}{q_{\max }-q_{\min }} & \text { if } q_{\min } \leqslant \bar{q}(k \delta) \leqslant q_{\max }, \\ 1 & \text { if } \bar{q}(k \delta) \geqslant q_{\max } .\end{cases}$

Mathematically, this queue length based rate combination scheme corresponds to a proportional controller plus lowpass filter. This relationship between $\gamma$ and $\bar{q}$ is also illustrated in Fig. 4. In terms of the choice of thresholds $q_{\min }$ and $q_{\text {max }}$, we consider the tradeoff between packet delay and smooth change of $\gamma$. In here, $q_{\min }$ and $q_{\max }$ are set to be 5 and 25, respectively. For the delay-guarantee design, we can replace this proportional controller by a proportional-integral controller in order to maintain the average queue length around the expected value. The non-cooperative rate combination algorithm is also illustrated in Fig. 5.

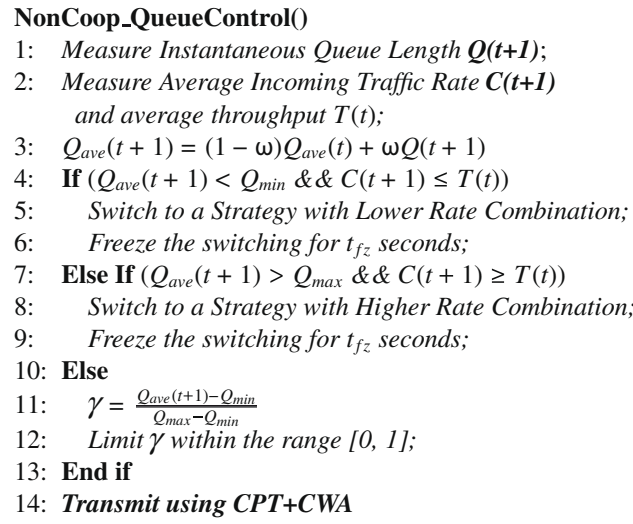

Fig. 5. Algorithm specification.

\subsection{Stability analysis}

In this subsection, we prove the stability of this proportional controller plus low-pass filter. The average probabilities in the collision, idle backoff and the successful transmission stages depend on contention window size of the users. Because the binary exponential backoff parameters at $r_{4}, r_{6}$ and $r_{8}$ are identical, each user has exactly the same $p_{s, i}, p_{d}$ and $p_{c, i}$. According to the expressions of throughput in Eqs. (26) and (27), each user's effective throughput is proportional to its average transmit rate. We made an additional assumption in order to simplify our analysis: (A5). The packet collisions, the exponential backoff scheme as well as the PHY/MAC overheads are not considered in the throughput model Eqs. (26) and (27), e.g. $p_{d}, p_{c, i}$ and $T_{o}$ are all zero. Note that this assumption is reasonable because the packet collision probability, backoff mechanism and the PHY/MAC overheads are independent of the physical data rates. Thus, a node's throughput is equivalent to the data rate over the number of active nodes. Without assumption (A5), the throughput in Eqs. (26) and (27) are still linear functions with respect to the average data rate. The difference only lies in that the proportional coefficient is smaller than the one (i.e. $\frac{1}{N}$ ) with (A5). To analyze this system, we consider a WLAN or a single-cell ad hoc network via fluid approximation. Therefore, network variables (e.g. queue length, throughput, data rate) are not discrete parameters, but rather, continuous functions of time.

Under the CPT/CWA scheme, the normalized airtimes of all saturated clients are the same (i.e. $\frac{1}{N}$ where $N$ is reused here to denote the number of clients in a cell). Based on the assumption A5, the throughput $T_{n}\left(x_{n}\right)$ of a client node can be expressed by,

$T_{n}\left(x_{n}(t)\right):=x_{n}(t) / N$.

The dynamic of instantaneous IFQ length can be described by,

$\dot{q}_{n}(t)= \begin{cases}{\left[C_{n}-T_{n}\left(x_{n}(t)\right)\right]^{-}} & \text {if } q_{n}(t)=q_{\max }, \\ C_{n}-T_{n}\left(x_{n}(t)\right) & \text { if } 0 \leqslant q_{n}(t) \leqslant q_{\max }, \\ \left.C_{n}-T_{n}\left(x_{n}(t)\right)\right]^{+} & \text {if } q_{n}=0,\end{cases}$

Fig. 4. Transmission rate controller. 
where $\dot{q}_{n}(t)$ denotes $\partial q_{n}(t) / \partial t[\cdot]^{+}$, represents the function $\max (\cdot, 0)$, and $[\cdot]^{-}$represents the function $\min (\cdot, 0)$. Similar to the method used in [12], we convert the Eq. (28) into a differential equation. A natural candidate is,

$\frac{d \bar{q}}{d t}=A \bar{q}(t)+B q(t)$.

In a sample control system, $\bar{q}\left(t_{k+1}\right)$ is given by

$\bar{q}\left(t_{k+1}\right)=e^{A\left(t_{k+1}-t_{k}\right)} q\left(t_{k}\right)-\int_{t_{k}}^{t_{k+1}} e^{A\left(t_{k+1}-\tau\right)} B d \tau q\left(t_{k}\right)$.

Comparing Eq. (33) with Eq. (28), we obtain

$A=-B=\frac{\log _{e}(1-\omega)}{\delta}$.

Therefore, one can use the following differential equation to describe the behavior of EWMA queue length,

$\frac{d \bar{q}_{n}(t)}{d t}=\frac{\log _{e}(1-\omega)}{\delta} \bar{q}_{n}(t)-\frac{\log _{e}(1-\omega)}{\delta} q_{n}(t)$.

Combining Eqs. (21), (30), (31) and (35) together, we obtain the single input single output (SISO) system dynamics of the non-cooperative rate combination scheme with $\mathrm{CPT} /$ CWA,

$d q_{n}(t) / d t=C_{n}-x_{n}(t) / N$,

Eq. (35),

$\gamma_{n}^{\prime}(t)=\frac{\bar{q}_{n}(t)-q_{\min }}{q_{\max }-q_{\min }}$,

$x_{n}(t)=\gamma_{n}^{\prime}(t) r_{j}+\left(1-\gamma_{n}^{\prime}(t)\right) r_{i}$.

In the fluid model, we regard the rate combination strategy to be fixed, and do not consider the switching effect of strategies. The reason is that the switching decision is strictly confined by three strong conditions: (1) the average queue length is greater than $q_{\max }$ or smaller than $q_{\text {min }}$; (2) the average incoming rate is no larger than or no less than the throughput; (3) consecutive switchings are not allowed in a short time interval. According to the above analysis, this fluid model is composed of linear differential equations, in which control theory can be introduced for performance evaluation. Consequently, we obtain insight of the feedback control system around operating points directly. An operating point is defined as a system state where the system differential equations are all zero. That is, $x_{n}^{*}=C_{n} / N, q_{n}^{*}$ and $\gamma_{n}^{\prime *}$ can be calculated correspondingly. We define linearized operating point to be $\left(\tilde{x}_{n}, \tilde{\bar{q}}_{n}, \tilde{\gamma}_{n}^{\prime}\right)$, where

$\tilde{x}_{n}=x_{n}-x^{*} ; \quad \tilde{\bar{q}}_{n}=\bar{q}_{n}-\bar{q}_{n}^{*} ; \quad \tilde{\gamma}_{n}^{\prime}=\gamma_{n}^{\prime}-\gamma_{n}^{\prime *}$.

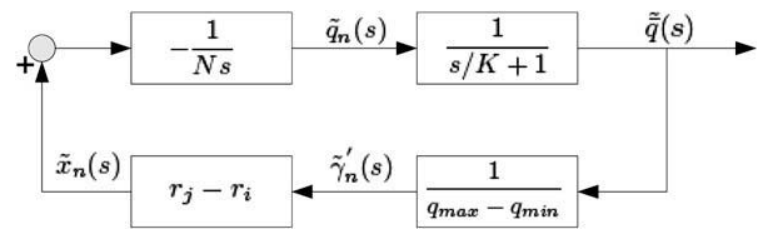

Fig. 6. Feedback control system block.
Using Laplace transformation, we obtain the frequency domain feedback control system block in Fig. 6 .

The open loop transfer function is expressed as

$G(s)=\frac{r_{j}-r_{i}}{N\left(q_{\max }-q_{\min }\right)} \cdot \frac{1}{s(s / K+1)}$

where $K$ is $-\frac{\log _{e}(1-\omega)}{\delta}$. Clearly one can see that $K$ is greater than 0 . Let the number of nodes, $N$, be within the range $\left\{N^{-}, N^{+}\right\}$and the traffic load of node $n$ is within the range $\left(\frac{x_{\min }}{N}, \frac{x_{\max }}{N}\right)$ Mbps, the close loop system is stable due to that both poles have negative real parts. Formally, we have the following proposition.

Proposition 3. The open loop linear feedback system in Eq. (37) with unit negative feedback is stable for all $N \in\left\{N^{-}, N^{+}\right\}$ and $x_{n} \in\left(\frac{x_{\min }}{N}, \frac{x_{\max }}{N}\right)$.

When the traffic demands of nodes $m,(m \in\{1,2, \ldots M\})$ are small enough so that they are not saturated even using the lowest rate $x_{\min }$ in their available combination set, the throughput of a saturated node $n, n \in\{M+1, \ldots, N\}$ can be calculated by

$T_{n}\left(x_{n}(t)\right)=\frac{x_{n}(t)\left(1-\sum_{m=1}^{M} C_{m} / x_{\min }\right)}{N-M}$

Similarly, we can also prove the stability for the saturated nodes whose traffic loads are within the feasible rate region.

If the packets are transmitted without CPT or CWA, the throughput of all saturated nodes are almost the same, that is

$T_{n}\left(x_{n}(t)\right):=\frac{1}{1 / x_{1}(t)+1 / x_{2}(t)+\cdots+1 / x_{N}(t)}$

Suppose there are $N$ client nodes in a WLAN whose demands satisfy $C_{1}<C_{2}<\cdots<C_{n}<\cdots<C_{N}$. If the demand $C_{1}$ is less than $x_{\min } / N$, the node 1 can transmit using data rate $x_{\min }$. If the demand $C_{1}$ is within the range $\left(\frac{x_{\min }}{N}, \frac{x_{\max }}{N}\right)$, we assume that the node 1 transmits using data rate $x_{1},\left(x_{1}>x_{\min }\right)$. This means that node 1 has backlogged packets in the interface queue. Thus, the throughput of nodes $2,3, \ldots N$ are less than their traffic demands. Only when the node 1 transmits using $x_{\min }$ and is unsaturated, the demands of remained nodes are likely to be served. The overriding disadvantage of rate combination scheme without CPT/CWA lies in that it drastically reduces the maximum throughput of a wireless network.

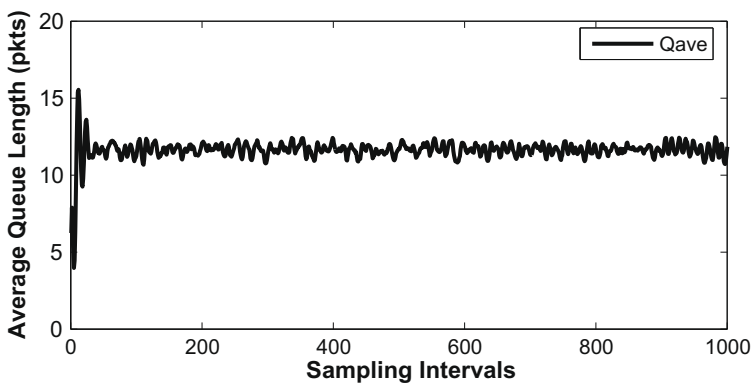

Fig. 7. Single link: avg. queue vs. sampling time. 


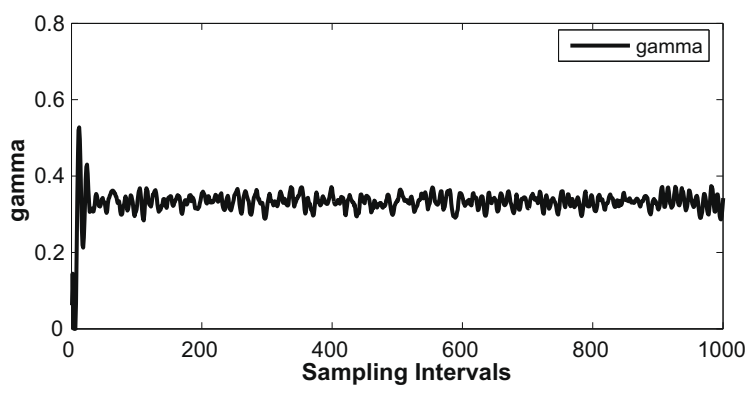

Fig. 8. Single link: rate prob. vs. sampling time.

\subsection{Numerical examples}

We illustrate the proposed non-cooperative algorithm through two representative numerical examples. The first one focues on the dynamic behavior of queue length and the probability of a single transmitting node, and the second one is on the interaction of two contending nodes with unequal traffic loads.

In the first experiment, the packet arrival rate follows the Poisson distribution with an average of 3000 packet/s and the packet length is 1000 bytes. The exponential weighting parameter $\omega$ is set to be 0.25 and the sampling interval is $0.01 \mathrm{~s}$. The maximum and minimum queue thresholds are set to be 25 and 5 packets by considering the tradeoff between sensitivity and robustness. Figs. 7 and 8 show that the queue length and probability $\gamma$ quickly converge to the equilibrium points. In the second numerical experiment, we set the average traffic demands to be 1800 packet/s and 1600 packet/s, respectively. The dynamics of average queue lengths and the probabilities are demonstrated in Figs. 9 and 10. To sum up, the proposed control system is not only stable, but also converges very fast in representative scenarios.

\section{Performance evaluation}

In this section, we evaluate both the dynamic performance and the energy efficiency of our proposal via ns2 simulations. Because standard 802.11 devices usually transmit using the highest achievable rate i.e. $54 \mathrm{Mbps}$, we compare our proposal with 802.11 standard whose transmission power is chosen to be the minimum value

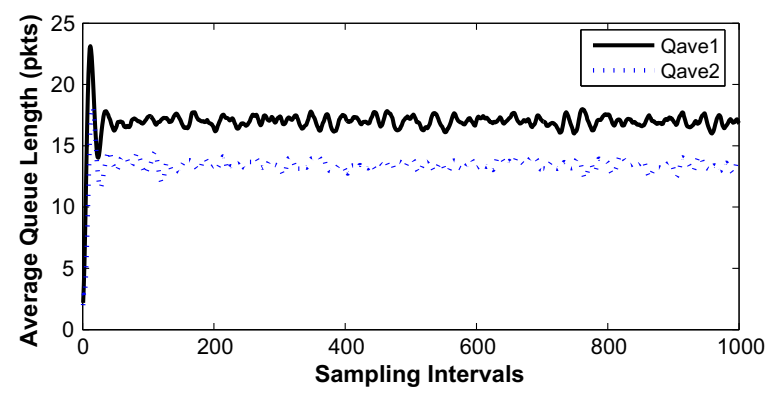

Fig. 9. Two links: avg. queue vs. sampling time.

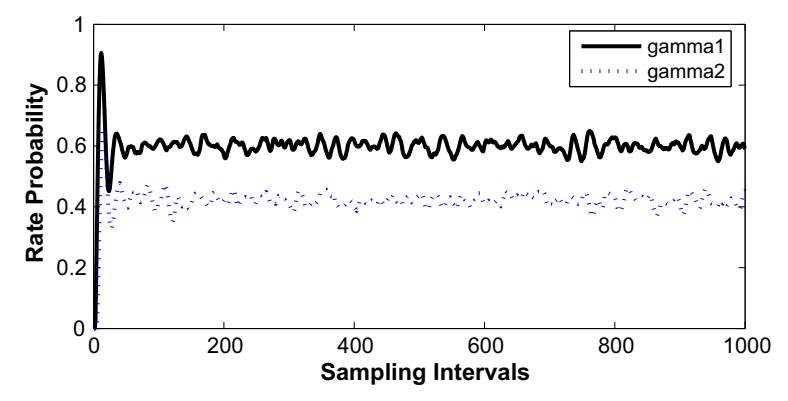

Fig. 10. Two links: rate prob. vs. sampling time.

of sustaining $54 \mathrm{Mbps}$ data rate. The antenna gains of transmitters and receivers are all set to 1 . The thermal noise is $-174 \mathrm{dbm} / \mathrm{Hz}$ and the bandwidth is $20 \mathrm{MHz}$. The path loss exponent is set to 3.1 in indoor environments according to the ITU recommendation. Other signal attenuations such as bricks and walls are $5 \mathrm{~dB}$. The maximum power of a transmitter is set to $1000 \mathrm{~mW}$. The PHY parameters of $802.11 \mathrm{a} / \mathrm{h}$ are chosen to be the same as those in $\mathrm{Kim}$ et al. [6]. In the proposed non-cooperative rate adaptation algorithm, we configure the system parameters as follows. The min and max thresholds are set to 5 packets and 25 packets, respectively. The incoming flow rate is sampled every one second and the queue length is measured in a much smaller time interval (0.005 $\mathrm{s}$ as default). The freeze time interval is $0.05 \mathrm{~s}$, which is merely to avoid consecutive switching between the combination strategies. The configurations of these timers work fine when the traffic measurement interval is the largest and the queue monitor interval is the smallest. The payload size of a flow is set to 1000 Bytes if it is not specified. In summary, the system parameters are listed in Table 4 as below.

\subsection{Dynamic behavior of probabilistic rate combination}

We evaluate the IFQ dynamics of two competing client nodes in this experiment. The average queue lengths of two sending nodes are shown in Fig. 11 when the traffic loads are both set to $8 \mathrm{Mbps}$. Because the loads are the same, the average queue lengths also fluctuate around almost the same value. Fig. 12 evaluates the queue dynamics of two transmitting nodes when flow 1 has a demand of $6 \mathrm{Mbps}$ and flow 2 has a demand of $10 \mathrm{Mbps}$. Both of the nodes choose the combination strategy $\{18,36\}$ Mbps. Thus, the average queue length of flow 2 is larger than that of flow 1 .

Table 4

Parameter table.

\begin{tabular}{llll}
\hline Parameter & Value & Parameter & Value \\
\hline Pathloss factor & 3.1 & Antenna gains & 1 \\
Thermal noise & $-174 \mathrm{dbm} / \mathrm{Hz}$ & Bandwidth & $20 \mathrm{MHz}$ \\
Other attenu. & $5 \mathrm{~dB}$ & Max trans. power & $1 \mathrm{~W}$ \\
Min threshold & $5 \mathrm{pkts}$ & Max threshold & $25 \mathrm{pkts}$ \\
Payload size & 1000 bytes & Queue monitor intv. & $0.005 \mathrm{~s}$ \\
Buffer size & 100 pkts & EWMA parameter & 0.25 \\
Measure intv. & $1 \mathrm{~s}$ & Freeze intv. & $0.05 \mathrm{~s}$ \\
\hline
\end{tabular}




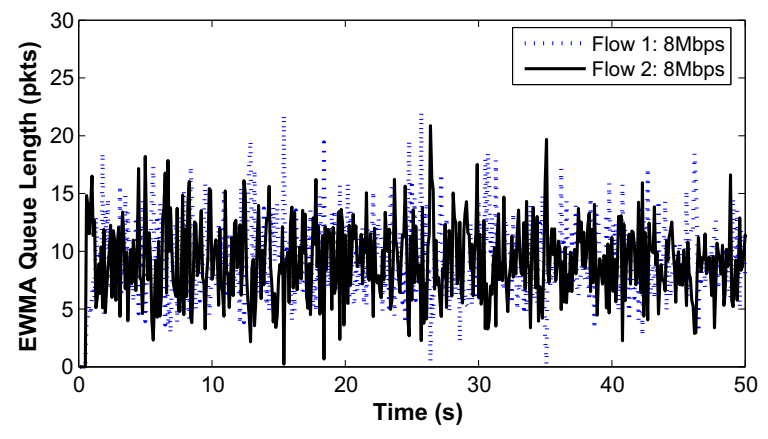

Fig. 11. Queue dynamics of homogeneous flows.

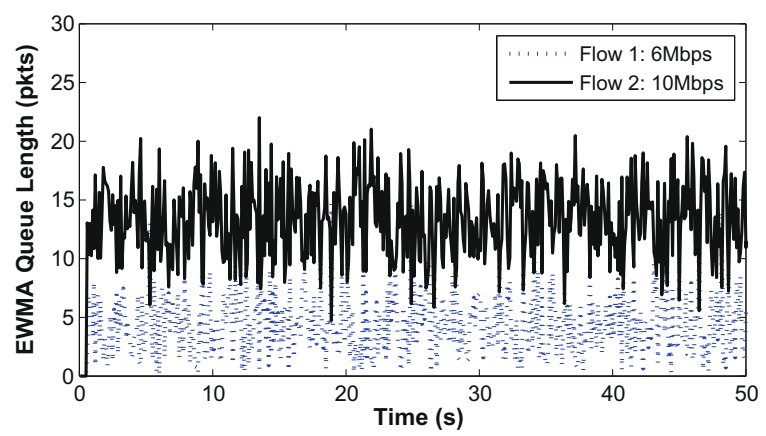

Fig. 12. Queue dynamics of heterogeneous flows.

\subsection{Distributed algorithm vs. centralized algorithm}

The energy efficiency problem can also be solved when the full information such as airtime and energy expenditure are available at each transmitter. By exhaustive search over the feasible data rates, one can identify the optimal transmission strategy. The centralized scheme requires the transmitters to collect the complete airtime and energy consumption information. Therefore, the centralized scheme has better energy gains than the our distributed algorithm in general. However, it is not easy to find the optimal transmission probability due to packet losses and computational complexity. The centralized scheme without probabilistic rate combination is usually worse than the proposed algorithm, even performs very poor in the presence of information cheating. We employ a simple experiment to validate our claim. Consider a wireless network composed of two transmission pairs, each of which has a traffic load of $8.5 \mathrm{Mbps}$. The hop distance of link 1 is $30 \mathrm{~m}$ and that of link 2 increases from $20 \mathrm{~m}$ to $40 \mathrm{~m}$. For fair comparison, we only calculate the power consumption of the transmitters because the energy of a receiver is independent of its transmitter. According to the previous analysis, the efficient rate set is $\{18,36,54\}$ Mbps. In each packet transmission, the percentage of payload airtime is around $79 \%$ for $18 \mathrm{Mbps}$, 65\% for $36 \mathrm{Mbps}$ and $55 \%$ for $54 \mathrm{Mbps}$. To transmit $8.5 \mathrm{Mbps}$ for a link, the needed airtime is $0.64 \mathrm{~s}$ for $18 \mathrm{Mbps}, 0.41 \mathrm{~s}$ for $36 \mathrm{Mbps}$ and $0.33 \mathrm{~s}$ for $54 \mathrm{Mbps}$ in one second. Hence, there are three efficient

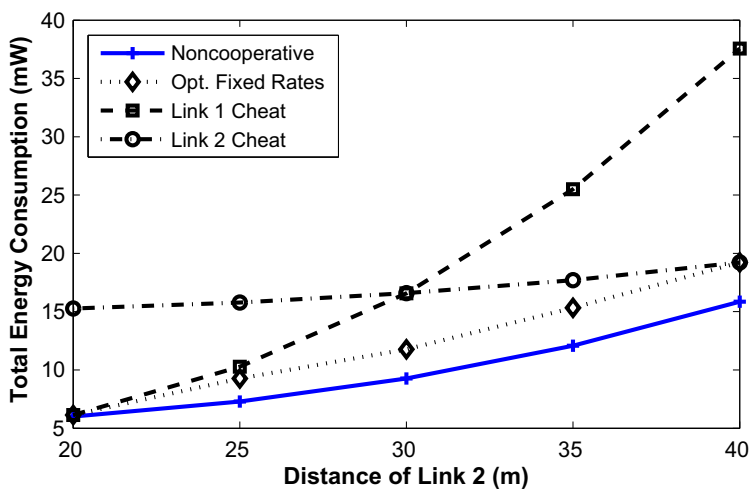

Fig. 13. Total energy expenditure with info. cheating.

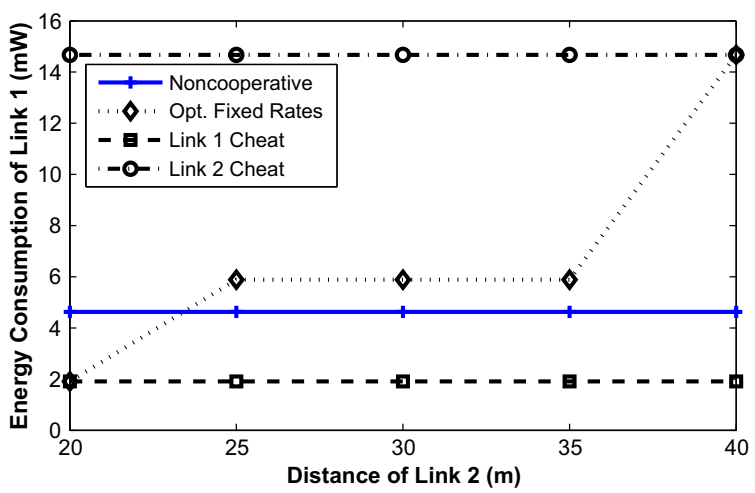

Fig. 14. Energy expenditure of the first transmitter.

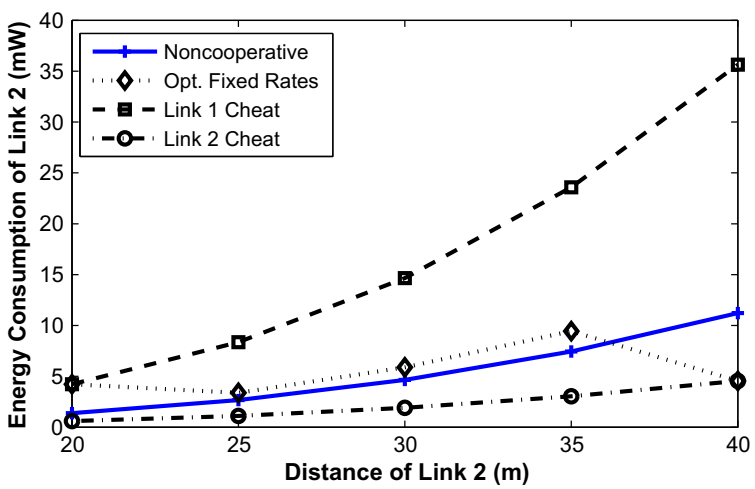

Fig. 15. Energy expenditure of the second transmitter.

rate combination strategies: $\{18,54\} \mathrm{Mbps},\{54,18\} \mathrm{Mbps}$ and $\{36,36\}$ Mbps. We compare the optimal fixed-rate combination scheme with the probabilistic rate combination scheme in Figs. 13-15. When the hop distance of link 2 increases from $20 \mathrm{~m}$ to $40 \mathrm{~m}$, the optimal fixed-rate combinations are chosen to be $\{18,54\},\{36,36\},\{36,36\},\{36,36\}$ and $\{54,18\}$. One can see in Fig. 13 that the proposed 
scheme has smaller energy expenditures than the optimal fixed-rate combination scheme. The individual energy consumptions of link 1 and 2 are also illustrated in Figs. 14 and 15. Because the centralized scheme relies on the information exchange between link 1 and 2, they can forge the values of energy expenditure. For example, link 1 cheats link 2 by reporting an arbitrarily large energy. Hence, link 2 transmits using $54 \mathrm{Mbps}$, no matter what the path loss is, while link 1 adopts 18 Mbps to reduce its energy expenditure. Figs. 13 and 15 show that the information cheating causes energy waste, especially when the hop distance of link 2 is large. Similarly, if link 2 reports fake information, it can greatly conserve energy when the hop distance is less than $40 \mathrm{~m}$. If both links cheat each other, the fixed-rate combination scheme might be $\{36,36\}$ Mbps, which still causes energy waste.

\subsection{Energy expenditure vs. traffic loads}

We evaluate power consumption of the proposed method in a single-cell ad hoc network with three transmission pairs. All of them have the same traffic demands and the hop distances are set to $25 \mathrm{~m}$ uniformly. We compare the energy consumptions of our proposal with the scheme that always uses $54 \mathrm{Mbps}$ for data packets. As mentioned earlier, the energy consumption is composed of two parts, one is that of payloads, the other is that of overheads. To highlight their difference, we evaluate the energy consumptions of payloads and whole packets separately. Fig. 16 shows the payload energy expenditure of the proposed algorithm and the $54 \mathrm{Mbps}$ scheme. When the traffic demands are small, the proposed algorithm can significantly reduce power consumption. Provided the traffic demand of a node in the range $[0.5,4] \mathrm{Mbps}$, the $54 \mathrm{Mbps}$ scheme results in over $700 \%$ energy expenditures than the non-cooperative rate adaptation algorithm. If the traffic demand is further increased, the energy gain shrinks until 1 . This is to say, when the traffic demands are large enough, the proposed algorithm enables 802.11 MAC to transmit using 54 Mbps data rate.

We measure the energy consumption of payloads and overheads for both transmitters and receivers in Fig. 17.

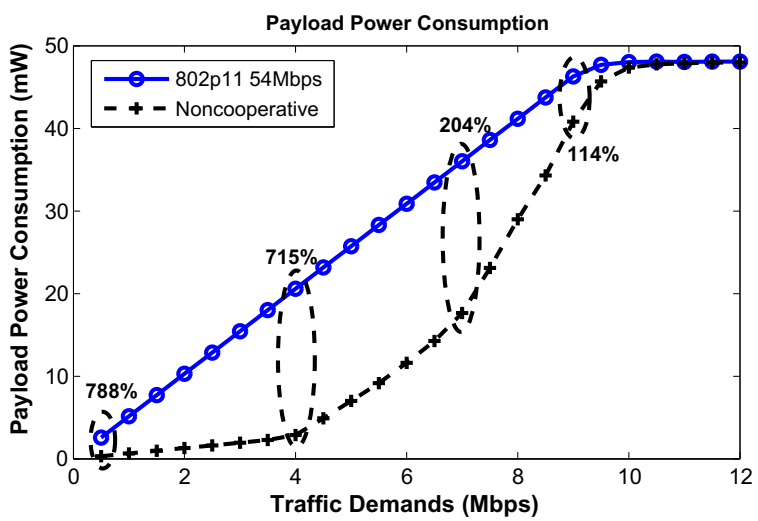

Fig. 16. Comparison of payload energy consumption.

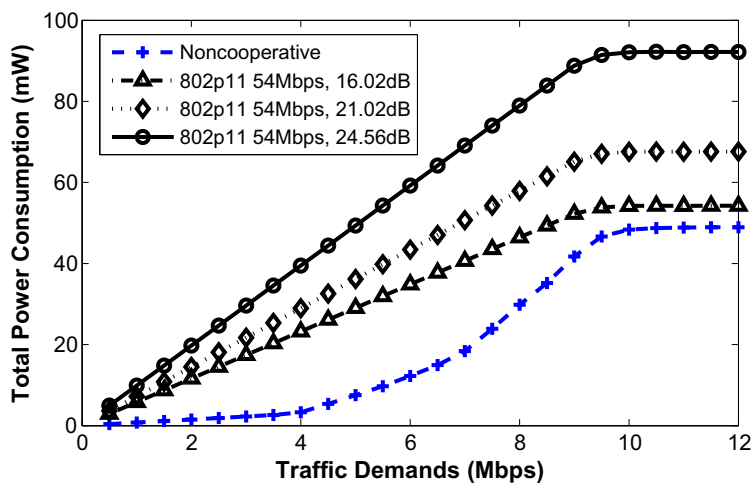

Fig. 17. Comparison of total energy consumption.

The rate of overheads is $6 \mathrm{Mbps}$ and the corresponding SNR threshold is $6.02 \mathrm{~dB}$. In the proposed algorithm, the power level of PHY/MAC overheads are $3 \mathrm{~dB}$ (or twice) higher than the minimum required one (i.e. $6.02 \mathrm{~dB}$ ). When the traffic loads are very heavy, the overheads merely consume less than $3 \%$ of total energy. For the $54 \mathrm{Mbps}$ scheme, we set three different overhead power levels that have SNRs of $16.02 \mathrm{~dB}, 21.02 \mathrm{~dB}$ and $24.56 \mathrm{~dB}$ in the receiver, respectively. Note that if the SNR of overheads is $24.56 \mathrm{~dB}$, their transmission power is the same as that of payloads. Because the overheads occupy about $20-40 \%$ of total airtime, high power leads to vast energy waste. One can see that the $54 \mathrm{Mbps}$ scheme consumes much more energy than the proposed scheme, even when the traffic demands are large enough.

\subsection{Energy expenditure in random scenarios}

In this subsection, we validate the proposed algorithm in two type of random scenarios. Ten set of experiments are conducted within each scenario, and there are eight

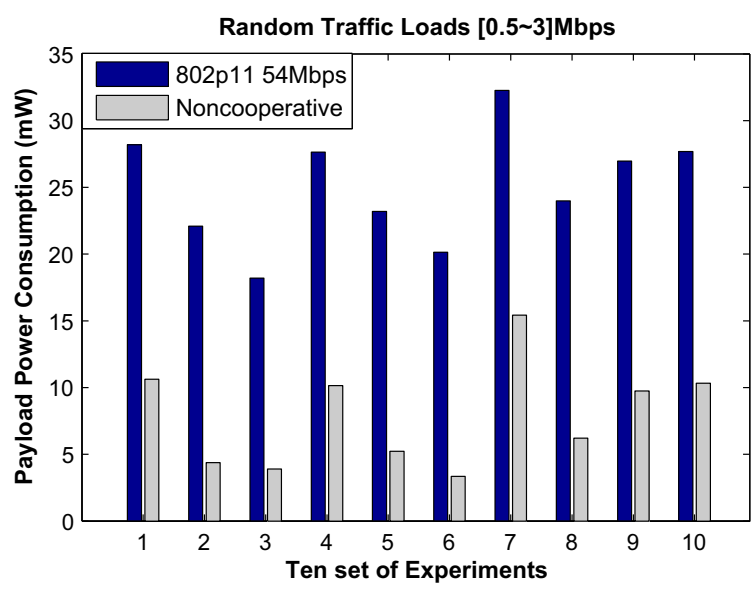

Fig. 18. Payload energy consumption in random scenarios. 


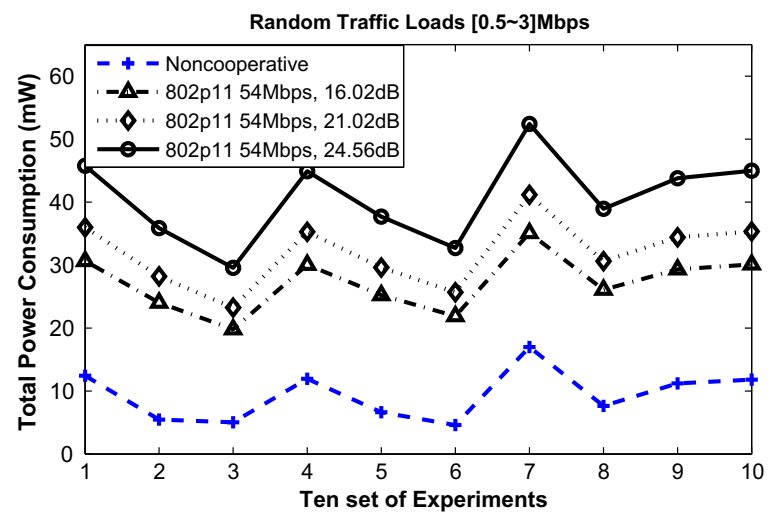

Fig. 19. Total energy consumption in random scenarios.

transmission pairs in every experiment. In the first type, we test 10 sets of eight randomly generated traffic demands that are within the range [0.5 Mbps, $3 \mathrm{Mbps}$ ]. The hop distances of all transmission pairs are all $30 \mathrm{~m}$ and the payload lengths are 1480 bytes uniformly. The payload

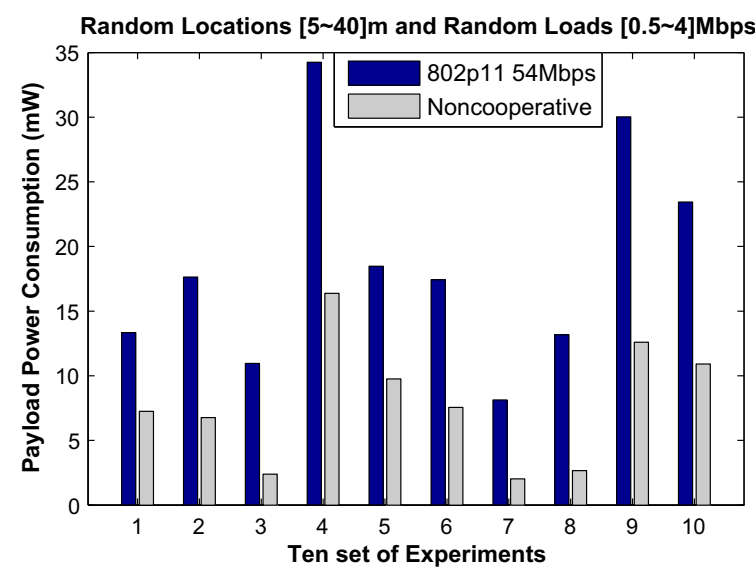

Fig. 20. Payload energy consumption in random scenarios.

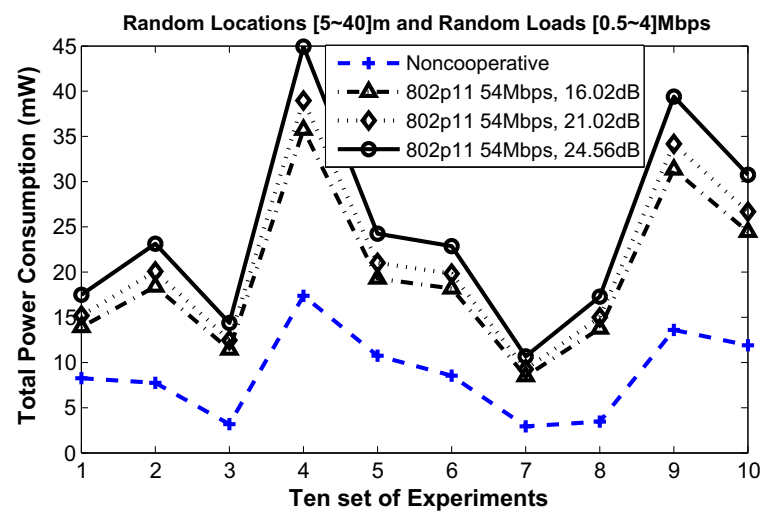

Fig. 21. Total energy consumption in random scenarios. energy consumptions of the proposed algorithm and the $54 \mathrm{Mbps}$ scheme are compared in Fig. 18. The energy gains range from $209 \%$ to $600 \%$ in these ten different traffic patterns. When the overheads are taken into consideration, the proposed algorithm can further reduce energy expenditure, which is demonstrated in Fig. 19.

Next, we evaluate the energy expenditure of nodes that are randomly distributed in the sites $[5,40] \mathrm{m}$ away from the access point and have traffic loads in the range $[0.5,4]$ Mbps. Similar to the preceding experiments, we record the payload energy consumption and the total energy consumption in Figs. 20 and 21, respectively. One can see that the proposed scheme significantly outperforms the $54 \mathrm{Mbps}$ scheme even when wireless nodes are randomly distributed in a WLAN or ad hoc network.

\section{Related work}

Previous energy efficient mechanisms focus on saving battery power by putting the wireless node in an idle state and there have been number of proposals in this direction. Transmission power control (TPC) is recognized as one of the most effective ways to reduce power consumption in 802.11 multi-rate wireless networks. Generally, the TPC and the physical rate adaptation are designed jointly to maximize link throughput and reduce power consumption. Here, we review two important algorithms that are closely related to our work.

Miser [4] computes an optimal rate-power configuration table in an offline mode, and then at runtime, a wireless station determines the most energy efficient transmission strategy for each data frame by a simple table lookup. But the rate-power strategy is only designed for saturated traffic, which is not applicable for dynamic traffic demands in a realistic wireless network. Another problem is that Miser algorithm needs a priori knowledge of the number of competing nodes.

Cooperative rate adaptation scheme (CRA) [5] is initially developed to save energy for a given traffic pattern in 802.11 multi-rate multi-hop ad hoc networks. It is an interesting work that targets at the energy conservation problem for a fixed traffic pattern. Under the CRA, each node exchanges the needed channel airtime of all rate levels with those nodes within their maximum interference range. However, CRA only considers the choice of data rates in an ideal multi-hop networks and might encounter following problems in realistic deployment: (1) It is difficult to construct a conflict graph for an arbitrary ad hoc network. In particular, different rate levels have very different interference ranges, the number of conflict graphs would increase exponentially. If the conflict graph is not correctly constructed, either the traffic demand might not be satisfied or the battery energy might be wasted. (2) The information exchange of required airtime uses the maximum transmission power and the lowest data rate in order to guarantee successful reception. Furthermore, the self-centric wireless nodes are inclined to exaggerate their energy expenditure so as to minimize the individual consumption. (3) The required airtime does not include the airtime wasted in packet collisions due to simulta- 
neous transmission. Our paper can be regarded as a distributed approach to solve a similar problem in which each user adjusts the transmit strategy on its own behalf.

\section{Conclusion}

In this paper, we show that probabilistic rate combination can greatly reduce power consumption and at the same time, is able to support a given traffic demand. The rate combination scheme is modeled as a non-convex optimization problem. We propose a non-cooperative rate combination algorithm to reduce power consumption. Each node adapts its rate strategy by observing the average IFQ length. Due to the "rate anomaly" of multi-rate wireless network, the nodes with smaller traffic demands have no incentive to increase their data rates. We introduce a joint consecutive packet transmission and contention window control to eliminate this problem. We also show the stability of our proposed method and illustrate its effectiveness via extensive simulations.

\section{References}

[1] S. Agarwal, S. Krishnamurthy, R. Katz, S. Dao, Distributed power control in ad-hoc wireless networks, in: Proceedings of IEEE PIMRC'01, 2001, pp. 59-66.

[2] J. Ebert, A. Wolisz, Combined tuning of RF power and medium access control for WLANs, ACM Mobile Networks and Applications 5 (6) (2001) 417-426.

[3] J. Gomez, A. Campbell, M. Naghshineh, C. Bisdikian, Conserving transmission power in wireless ad hoc networks, in: Proceedings of IEEE ICNP'01, 2001, pp. 24-34.

[4] D.J. Qiao, S. Choi, A. Jain, K. Shin, MiSer: an optimal low-energy transmission strategy for IEEE $802.11 \mathrm{a} / \mathrm{h}$, in: Proceedings of ACM Mobicom'03, San Diego, USA, 2003, pp. 14-19.

[5] K. Wang, F. Yang, Q. Zhang, et al, Distributed cooperative rate adaptation for energy efficiency in IEEE 802.11-based multi-hop networks, IEEE Transactions on Vehicular Technology (2007). March.

[6] T.S. Kim, J.C. Hou, H. Lim, Improving spatial reuse through tuning transmit power, carrier sense threshold, and data rate in multihop wireless networks, in: Proceedings of ACM Mobicom'06, Los Angeles, USA, 2006, pp. 366-377.

[7] D.J. Qiao, K.G. Shin, Z. Abichar, Energy conservation in 802.11 WLANs via transmission strategy-aware airtime allocation, Elsevier Computer Networks 52 (12) (2008) 2280-2291.

[8] M. Heusse, F. Rousseau, G.B. Sabbatel, A. Duda, Performance anomaly of $802.11 \mathrm{~b}$, in: Proceedings of IEEE Infocom'03, San Francisco, USA, 2003, pp. 836-843.

[9] V. Ramaiyan, A. Kumar, E. Altman, Fixed point analysis of single cell IEEE 802.11e WLANs: uniqueness, multistability and throughput differentiation, in: Proceedings of ACM Sigmetrics'05, Banff, Canada, 2005, pp. 109-120.

[10] G. Bianchi, Performance analysis of the IEEE 802.11 distributed coordination function, IEEE Journal on Selected Areas in Communications 18 (3) (2000) 535-547.

[11] S. Floyd, V. Jacobson, Random early detection gateways for congestion avoidance, IEEE/ACM Transactions on Networking 1 (4) (1993) 297-413.

[12] V. Misra, W. Gong, D. Towsley, A fluid-based analysis of a network of AQM routers supporting TCP flows with an application to RED, in: Proceedings of ACM SIGCOMM'00, vol. 30 (4), 2000, pp. 151-160.

[13] J. Yee, P.E. Hossain, Understanding wireless LAN performance tradeoffs, CommsDesign.com (2002).

[14] M. Lacage, M.H. Manshaei, T. Turletti, IEEE 802.11 rate adaptation: a practical approach, in: Proceedings of ACM MsWim'04, Venice, Italy, 2004, pp. 126-134.

[15] S.H.Y. Wong, S.W. Lu, H. Yang, V. Bharghavan, Robust rate adaptation for 802.11 wireless networks, in: Proceedings of ACM Mobicom'06, Los Angeles, USA, 2006, pp. 146-157.
[16] G. Holland, N. Vaidya, P. Bahl, A rate-adaptive MAC protocol for multi-hop wireless networks, in: Proceedings of ACM Mobicom'01, Rome, Italy, 2001, pp. 236-251.

[17] J. Kim, S. Kim, S. Choi, D. Qiao, CARA: Collision-aware rate adaptation for IEEE 802.11 WLANs, in: Proceedings of IEEE Infocom'06, Barcelona, Spain, 2006

[18] B. Sadeghi, V. Kanodia, A. Sabharwal, E. Knightly, Opportunistic media access for multirate ad hoc networks, in: Proceedings of IEEE Mobicom'02, New York, USA, 2002, pp. 24-35.

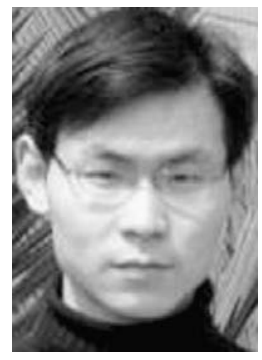

Yuedong $\mathbf{X u}$ is a Ph.D candidate in the Department of Computer Science and Engineering in the Chinese University of Hong Kong. He received his B.S. degree from Anhui University (AHU) in 2001 and M.S. from Huazhong University of Science \& Technology (HUST) in 2004 with honor, both in Control Science \& Engineering. His current interests lie in the control and optimization of wired and wireless networks.

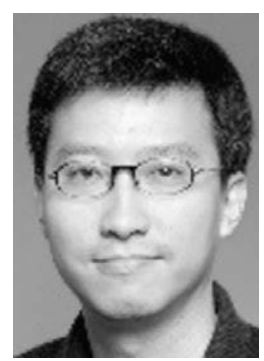

John C.S. Lui received his Ph.D in Computer Science from UCLA. He worked in the IBM San Jose/Almaden Research Laboratory before joining the Chinese University of Hong Kong. Currently, he is the chair of the Computer Science \& Engineering Department at CUHK and he leads the Advanced Networking \& System Research Group. His research interests span both in systems as well as in theory/mathematics in computer communication systems. John received various departmental teaching awards and the CUHK Vice-Chancellor's Exemplary Teaching Award, as well as the corecipient of the Best Student Paper Awards in the IFIP WG 7.3 Performance 2005 and the IEEE/IFIP Network Operations and Management (NOMS) Conference.

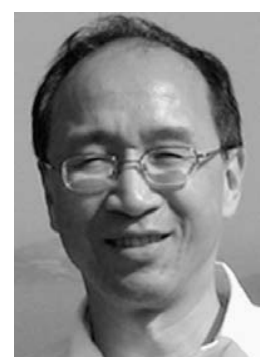

Dah-Ming Chiu, a Fellow of IEEE, received the B.Sc degree in Electrical Engineering from Imperial Colleage, University of London, and the Ph.D degree from Harvard University, in 1975 and 1980 respectively. He was a Member of Technical Staff with Bell Labs from 1979 to 1980 . From 1980 to 1996 , he was a Principal Engineer, and later a Consulting Engineer at Digital Equipment Corporation. From 1996 to 2002, he was with Sun Microsystems Research Labs. Currently, he is a professor in the Department of Information Engineering in The Chinese University of Hong Kong. He is known for his contribution in studying network congestion control as a resource allocation problem, the fairness index, and analyzing a distributed algorithm (AIMD) that became the basis for the congestion control algorithm in the Internet. His current research interests include economic issues in networking, P2P networks, network traffic monitoring and analysis, and resource allocation and congestion control for the Internet with expanding services. Two recent papers he co-authored with students have won best student paper awards from the IFIP Performance Conference and the IEEE NOMS Conference. Recently, Dr Chiu has served on the TPC of IEEE Infocom, IWQoS and various other conferences. He is a member of the editorial board of the IEEE/ACM Transactions on Networking, and the International Journal of Communication Systems (Wiley). 